\title{
A review of higher order strain gradient theories of plasticity: Origins, thermodynamics and connections with dislocation mechanics
}

\author{
SUMAN GUHA ${ }^{1}$, SANDEEP SANGAL $^{1}$ and SUMIT BASU ${ }^{2, *}$ \\ ${ }^{1}$ Department of Materials Science and Engineering, Indian Institute of Technology \\ Kanpur, Kanpur 208016, Uttar Pradesh, India \\ ${ }^{2}$ Department of Mechanical Engineering, Indian Institute of Technology Kanpur, \\ Kanpur 208016, Uttar Pradesh, India \\ e-mail: gsuman@iitk.ac.in; sangals@iitk.ac.in; sbasu@iitk.ac.in
}

MS received 19 April 2013; revised 06 May 2014; accepted 08 January 2015

\begin{abstract}
In this paper we review developments in higher order strain gradient theories. Several variants of these theories have been proposed in order to explain the effects of size on plastic properties that are manifest in several experiments with micron sized metallic structures. It is generally appreciated that the size effect arises from the storage of geometrically necessary dislocations (GNDs) over and above the statistically stored dislocations (SSDs) required for homogeneous deformations. We review developments that show that the GNDs result from the non-homogeneous nature of the deformation field. Though the connection between GNDs and strain gradients are established in the framework of single crystal plasticity, generalisations to polycrystal plasticity has been made. Strain gradient plasticity inherently involves an intrinsic length scale. In our review, we show, through a few illustrative problems, that conventional plasticity solutions can always be reduced to a scale independent form. The same problems are solved with a simple higher order strain gradient formulation to capture the experimentally observed size effects. However, higher order theories need to be thermodynamically consistent. It has recently been shown that only a few of the existing theories pass this test. We review a few that do. Higher order theories require higher order boundary conditions that enable us to model effects of dislocation storage at impermeable boundaries. But these additional boundary conditions also lead to unique conceptual issues that are not encountered in conventional theories. We review attempts at resolving these issues pertaining to higher order boundary conditions. Finally, we review the future of such theories, their relevance and experimental validation.
\end{abstract}

Keywords. Size effects; higher order strain gradient plasticity; geometrically necessary dislocations; thermodynamic consistency; higher order boundary conditions.

*For correspondence 


\section{Introduction}

In recent years there has been a surge of research in mechanics of materials at micron and sub-micron scales. With the advent of advanced tools to explore and manipulate structures at these scales, it is now possible to manipulate extremely small samples and test the behavior of materials at these scales. These studies have revealed that the material behavior is different at micron and lower scales compared to the bulk. In general, it is understood that the strength of materials is notably higher at scales of microns or lower. This is commonly known as the size effect. Well-known applications of this phenomenon are in strengthening of materials by grain refinement or by dispersion hardening. The strength of the material in these cases is improved by reducing the size of the microstructural constituents of the materials, namely, the grain size or the size of second phase particles. These effects (of which the well-known Hall-Petch effect is an example, Hall 1951; Petch 1953) are well described by dislocation theories for crystalline materials.

A somewhat different example of size effect is commonly observed in nanoindentation experiments. Here, the measured hardness scales with the depth of indentation, with the hardness being higher at smaller depth and gradually approaching the bulk hardness of the material as the depth of indentation increases (Nix \& Gao 1998). Moreover, a range of recent experimental studies have shown that, distinct from the well-known Hall-Petch type hardening, size effects are associated simply with the plastic deformation of micron-sized structures (Motz et al 2005; Fleck et al 1994; Stölken \& Evans 1998). These findings lend credence to the fact that "smaller is stronger" in the sense that when a structure has dimensions in the range of microns the material seems to exhibit significantly elevated yield stress. This is observed mostly in cases where the deformation is spatially non-uniform e.g., bending of thin beams (Motz et al 2005), torsion of thin wires (Fleck et al 1994), nanoindentations (Nix \& Gao 1998; Swadener 2002; McElhaney 1998), and micro-moulding (Ke 2011). Interestingly, similar apparent hardening behavior has also been recently observed in cases involving apparently uniform deformation e.g. micro-compression or micro-tension experiments (Uchic \& Dimiduk 2005; Budiman et al 2008a). Clearly, there is significant experimental evidence to suggest that materials behave differently at small scales. In particular, their plastic response seems to include dependence on sample size contrary to conventional theories of plasticity. It should be noted that strain gradient plasticity probably does not apply to situations at the scales at which the above experiments were conducted. In these cases, the number of dislocations in the specimens is small and the discrete nature of the dislocations comes into the picture. Continuum plasticity treats dislocations as distributions which manifest through the plastic strain or strain gradient.

In this paper we will review recent developments on theories of plasticity that seek to explain size dependence of mechanical properties in a continuum setting. Typically, these theories have intrinsic length scales built in and are designed to explain plastic deformation of small structures more realistically.

The development of newer theories of plasticity incorporating intrinsic length scales - christened "strain gradient plasticity" for reasons that will soon become apparent - is by no means either complete or without controversies. In this review, we will trace out its development using the following route. In the next section, we will illustrate the basic idea behind scale dependent solutions to problems. In section 3, scale-free solutions are obtained to a few simple problems using the conventional deformation theory of plasticity. These solutions are compared to experimental results at small scales in order to underline the need for a theory with a length scale. Section 4 samples ideas from the theory of dislocations and crystal plasticity to 
establish that gradients of plastic strain are associated with the concept of geometrically necessary dislocations (GNDs). Moreover, gradient of strain is an appropriate vehicle that allows a length scale to be introduced, thereby tracing the origin of the size effect to the existence of GNDs. In section 5, a continuum mechanics approach is adopted to establish a thermodynamic framework for strain gradient theories and well-known theories are positioned within this framework. We use one of the simplest forms of the theory of strain gradient plasticity to solve a few problems with a view to demonstrate the description of size effects. Issues regarding boundary conditions required for higher order strain gradient theories are discussed in section 6. Finally, we end by discussing our views regarding the future of these theories of plasticity.

\section{Nonlocal theory - introduction of a length scale}

Classical continuum theories are based on the assumption that stresses at a material point are expressed in terms of strains (and possibly other quantities) only at that point. Theories based on such a definition of a constitutive law are usually known as local (Bažant \& Jirásek 2002). On the contrary if the neighbourhood of a point also influences the mechanical response of the material at that point, the constitutive relations require modifications. These modifications are usually

achieved either by an integral averaging (e.g. $\sigma(x)=\int_{-\infty}^{\infty} E(x, p) \epsilon(p) d p$ ) or by introducing a gradient term to the existing constitutive equation. The resulting laws are termed as nonlocal. We are specifically interested in the latter case. For example

$$
\sigma(x)=E \epsilon(x)
$$

is a simple local constitutive law. A local constitutive law results in a scale independent governing equation. Combining the equilibrium equation, the above constitutive relation and the strain displacement relation for a one-dimensional problem shown in figure 1 generate a governing equation,

$$
E \frac{d^{2} u}{d x^{2}}+b=0
$$

where $b$ is the body force per unit volume and $u$ is the displacement. The above equation is a second order ordinary differential equation. The scale invariance of this equation is easily established by scaling the space coordinates by a factor $\beta$, i.e. $\bar{x}=\beta x$ and $\bar{u}=\beta u$. In the scaled coordinates the differential equation retains its form to become,

$$
E \frac{d^{2} \bar{u}}{d \bar{x}^{2}}+\bar{b}=0
$$

where $\bar{b}=b / \beta$. Note that the governing equation for the bar has exactly the same form in the scaled and original coordinate systems.

Now, consider that the stress depends on $d \epsilon / d x$ as well, in which case,

$$
\sigma(x)=E\left[\epsilon(x)+l_{*} \frac{d \epsilon(x)}{d x}\right],
$$




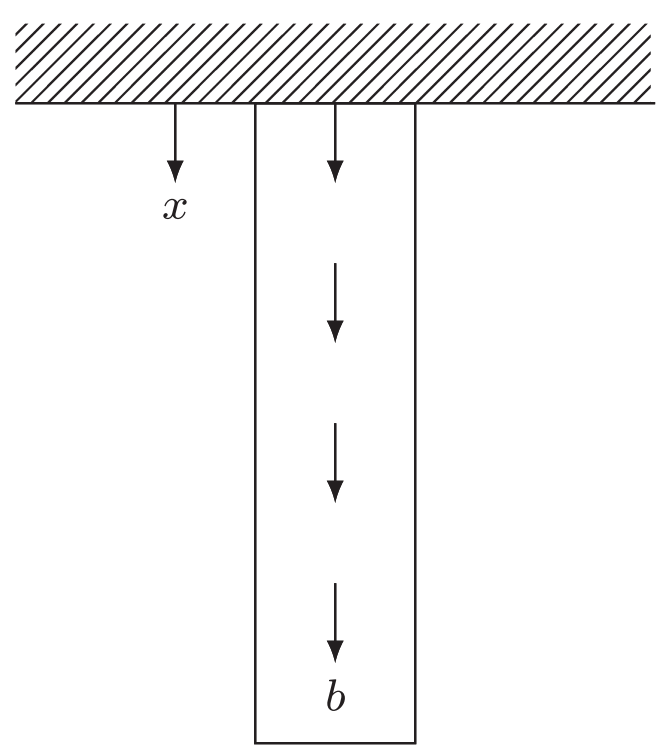

Figure 1. One-dimensional bar under body force.

where $l_{*}$ is a constant having dimension of length and has to be introduced for dimensional consistency. The governing differential equation for the one-dimensional problem figure 1 now becomes,

$$
E \frac{d^{2} u}{d x^{2}}+E l_{*} \frac{d^{3} u}{d x^{3}}+b=0 .
$$

The above equation, unlike Eq. (3), is not scale invariant. Substituting $\bar{x}=\beta x$ and $\bar{u}=\beta u$ gives

$$
E \frac{d^{2} \bar{u}}{d \bar{x}^{2}}+\beta E l_{*} \frac{d^{3} \bar{u}}{d \bar{x}^{3}}+\bar{b}=0,
$$

which does not have the same form as Eq. (5). Such constitutive models are termed nonlocal and $l_{*}$ serves as the parameter that introduces size dependence.

While Eq. (5) can be easily solved, the solution will be dependent on $l_{*}$, the length scale in the problem. If the length of the bar is $L$, and $L / l_{*}>>1$, the solution will tend towards that given by figure 1 . However, for $L / l_{*}<1$, the solution will differ significantly.

This example, though not based on a problem in plasticity, allows us to demonstrate the concept of scale invariance. Moreover, the problem illustrates that even in the case of linear elasticity, introducing a dependence of the stress on a higher order derivative of the displacement, leads to scale dependence. If a physical problem is governed by scale invariant differential equations (as in figure 1), a solution obtained for a large domain with some boundary conditions will be applicable for a scaled down small domain under the similar boundary conditions, irrespective of how drastic the scaling is. It is easy to imagine that the scaling will fail if the large domain is miniaturised to atomistic scales and the continuum assumptions, under which the governing differential equation has been formulated, fails. However, experimental results seem to suggest that the scale invariance of problems in continuum mechanics breaks down at scales much larger than the atomistic - probably in the range of tens of microns. For example, indentation size effects manifest at depths less than a few microns (Nix \& Gao 1998), thin shafts of diameter around 
$25 \mu \mathrm{m}$ and beams of similar thickness seem to be stronger than their bulk counterparts (Fleck \& Hutchinson 1997; Stölken \& Evans 1998). We will now show that problems in conventional plasticity are necessarily scale invariant.

\section{Few problems in classical plasticity}

\subsection{Basic kinematics}

For plastic deformation in a polycrystalline material, it is assumed that the lattice undergoes plastic deformation by dislocation slips only, while the lattice structure and orientation remains unchanged. This is followed by an elastic stretching and rotation of the lattice (see figure 2). If the motion of the body is defined as a linear mapping of a material point $X_{i}$ to $x_{i}$ in the deformed configuration according to,

$$
\boldsymbol{x}=\chi(\boldsymbol{X}), \text { and } \boldsymbol{u}=\boldsymbol{x}-\boldsymbol{X},
$$

the deformation gradient tensor $\boldsymbol{F}$ is,

$$
\boldsymbol{F}=\nabla_{X} \boldsymbol{x}, \text { and } J=\operatorname{det} \boldsymbol{F}>0,
$$

where $\nabla_{\boldsymbol{X}}=\partial / \partial \boldsymbol{X}$ and $\operatorname{det}(\cdot)$ is the determinant. Also, we define the velocity gradient tensor $\boldsymbol{L}$ as,

$$
\boldsymbol{L}=\nabla \dot{\boldsymbol{u}}=\dot{\boldsymbol{F}} \boldsymbol{F}^{-1} .
$$

Plastic deformation convects a material element vector $d \boldsymbol{X}$ to a vector $d \overline{\boldsymbol{X}}$ at an intermediate configuration according to,

$$
d \overline{\boldsymbol{x}}=\boldsymbol{F}^{p} d \boldsymbol{X} .
$$

Thereafter it undergoes elastic stretching and rotation and convects to $d \boldsymbol{x}$ in the deformed configuration according to,

$$
d \boldsymbol{x}=\boldsymbol{F}^{e} d \overline{\boldsymbol{x}} .
$$

It is also easy to show that,

$$
\boldsymbol{F}=\boldsymbol{F}^{e} \boldsymbol{F}^{p} \text { and } J=J^{e} J^{p} .
$$

Also,

$$
\begin{aligned}
\boldsymbol{L} & =\boldsymbol{L}^{e}+\boldsymbol{F}^{e} \boldsymbol{L}^{p} \boldsymbol{F}^{e-1}, \quad \text { where } \\
\boldsymbol{L}^{e} & =\dot{\boldsymbol{F}}^{e} \boldsymbol{F}^{e-1} \text { and } \boldsymbol{L}^{p}=\dot{\boldsymbol{F}}^{p} \boldsymbol{F}^{p-1} .
\end{aligned}
$$

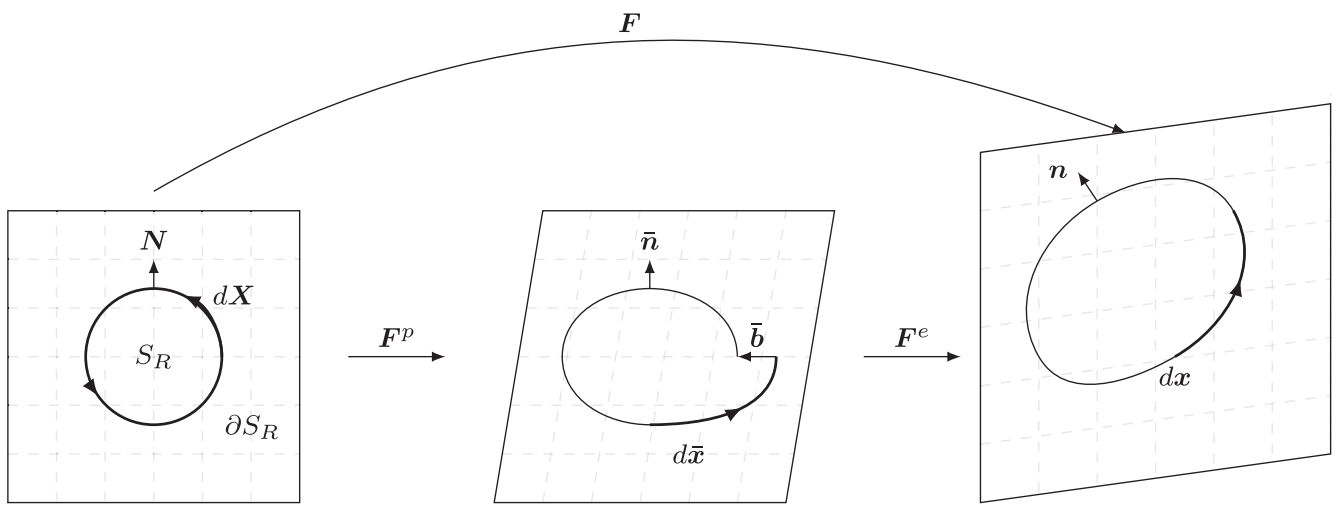

Figure 2. Schematic representation of lattice shear due to slip, followed by elastic stretching and rotation. 
Here, $J^{e}=\operatorname{det}\left(\boldsymbol{F}^{e}\right)$ and $J^{p}=\operatorname{det}\left(\boldsymbol{F}^{p}\right)$.

Further, the elastic and plastic stretch and spin tensors are defined as,

$$
\begin{aligned}
& \boldsymbol{D}^{e}=\frac{1}{2}\left(\boldsymbol{L}^{e}+\boldsymbol{L}^{e T}\right), \quad \boldsymbol{D}^{p}=\frac{1}{2}\left(\boldsymbol{L}^{p}+\boldsymbol{L}^{p T}\right), \\
& \boldsymbol{W}^{e}=\frac{1}{2}\left(\boldsymbol{L}^{e}-\boldsymbol{L}^{e T}\right), \quad \boldsymbol{W}^{p}=\frac{1}{2}\left(\boldsymbol{L}^{p}-\boldsymbol{L}^{p T}\right),
\end{aligned}
$$

or as symmetric and anti-symmetric components of the corresponding velocity gradient tensors. As in the small deformation theory, it is further assumed that

$$
\operatorname{tr} \boldsymbol{D}^{p}=\mathbf{0}, \text { or } \operatorname{det} \boldsymbol{F}^{p}=\boldsymbol{I},
$$

which leads to the fact that,

$$
J=J^{e}=\operatorname{det} \boldsymbol{F}=\operatorname{det} \boldsymbol{F}^{e} .
$$

Strain tensors $\boldsymbol{E}^{e}$ and $\boldsymbol{E}^{p}$ are defined as,

$$
\begin{aligned}
\boldsymbol{E}^{e} & =\frac{1}{2}\left(\boldsymbol{F}^{e T} \boldsymbol{F}^{e}-\boldsymbol{I}\right), \text { and } \\
\boldsymbol{E}^{p} & =\frac{1}{2}\left(\boldsymbol{F}^{p T} \boldsymbol{F}^{p}-\boldsymbol{I}\right) .
\end{aligned}
$$

Moreover, it will be useful to note that,

$$
\operatorname{Curl}(\boldsymbol{F})=\nabla_{\boldsymbol{X}} \times \boldsymbol{F}=\nabla_{\boldsymbol{X}} \times\left(\nabla_{\boldsymbol{X}} \boldsymbol{x}\right)=0
$$

where $\nabla_{X} \times(\cdot)$ is the curl of the quantity $(\cdot)$ with respect to reference configuration defined as,

$$
\left(\nabla_{\boldsymbol{X}} \times \boldsymbol{F}\right)_{i j}=\varepsilon_{i r s} \frac{\partial F_{j s}}{\partial X_{r}} .
$$

However, $\operatorname{Curl}\left(\boldsymbol{F}^{p}\right)$ and $\operatorname{Curl}\left(\boldsymbol{F}^{e}\right)$ individually are not equal to zero in general. In fact, the incompatibility in plastic strain could be related directly to $\operatorname{Curl}\left(\boldsymbol{F}^{p}\right)$.

\subsection{Problems in classical plasticity}

Classical plasticity is essentially a local theory and cannot model size effects. In order to contrast results from strain gradient plasticity, a few representative problems are solved in this section with conventional deformation theory of plasticity. The problems will be solved under the simplifying assumption that $\boldsymbol{F}^{e}=\boldsymbol{I}$. In particular, we will demonstrate that conventional plasticity solutions can always be reduced to scale independent forms. Further, for all subsequent analyses the deformation theory approach is undertaken under the assumption of infinitesimal deformations on a body $V$ with its surface denoted by $\partial V$. The developments closely follow (Chakraborty 2006). The uniaxial response of the material follows a power law relation according to,

$$
\sigma_{e}=\sigma_{0}\left(\frac{\epsilon_{e}}{\epsilon_{0}}\right)^{n},
$$

where $\epsilon_{0}$ is a reference strain, $\sigma_{0}$ the yield stress and $n$ the strain hardening exponent. The conventional equilibrium equation and the boundary conditions are

$$
\begin{aligned}
\sigma_{j i, j} & =0 \text { on } V, \text { and } \\
T_{i} & =\sigma_{j i} n_{j} \text { on } \partial V,
\end{aligned}
$$


where $\sigma_{i j}$ is the Cauchy stress tensor and $T_{i}$ is the surface traction vector on the boundary $\partial V$ with unit normal $n_{j}$. In deformation theory of conventional plasticity, there is no formal distinction between plastic and elastic parts of the strain and the material behavior is similar to a non-linear elastic material. Additionally, the material is considered to be incompressible and hence $\epsilon_{k k}=0$. The incremental work of deformation is balanced by the increment in the stored energy according to,

$$
\delta W_{I}=\sigma_{i j} \delta \epsilon_{i j}=\sigma_{i j}^{\prime} \delta \epsilon_{i j}=\sigma_{e} \delta \epsilon_{e},
$$

where $\sigma_{i j}^{\prime}$ is the deviatoric part of $\sigma_{i j}$ defined as,

$$
\sigma_{i j}=\sigma_{i j}^{\prime}+\frac{1}{3} \delta_{i j} \sigma_{k k}
$$

The equivalent stress $\sigma_{e}$ and equivalent strain $\epsilon_{e}$ are defined in the usual manner as

$$
\sigma_{e}=\sqrt{\frac{3}{2} \sigma_{i j}^{\prime} \sigma_{i j}^{\prime}}, \text { and } \epsilon_{e}=\sqrt{\frac{2}{3} \epsilon_{i j} \epsilon_{i j}}
$$

The constitutive law for the deviatoric part $\sigma_{i j}^{\prime}$ can be derived from (25) as,

$$
\sigma_{i j}^{\prime}=\frac{\partial W_{I}}{\partial \epsilon_{i j}}=\frac{2 \sigma_{e}}{3 \epsilon_{e}} \epsilon_{i j}
$$

Using this standard formulation we derive results for a few simple problems in order to show that classical plasticity predicts scale independent deformation behaviour. The results are derived in some detail as we will contrast them with results from strain gradient plasticity in section 5.4.

\subsection{Pure bending of a thin beam}

A thin beam of thickness $2 h$ and width $b$ is subjected to pure bending as shown in figure 3 . The beam is under symmetric bending, i.e., the neutral axis passes through $x_{2}=0$. In this case, the lateral strain in the beam is given by

$$
\epsilon_{11}=\kappa x_{2},
$$

where $\kappa$ is the curvature at the neutral axis of the beam. Material incompressibility demands that $\epsilon_{22}=-\epsilon_{11}=-\kappa x_{2}$ and all other components of the strain tensor are zero. Integrating

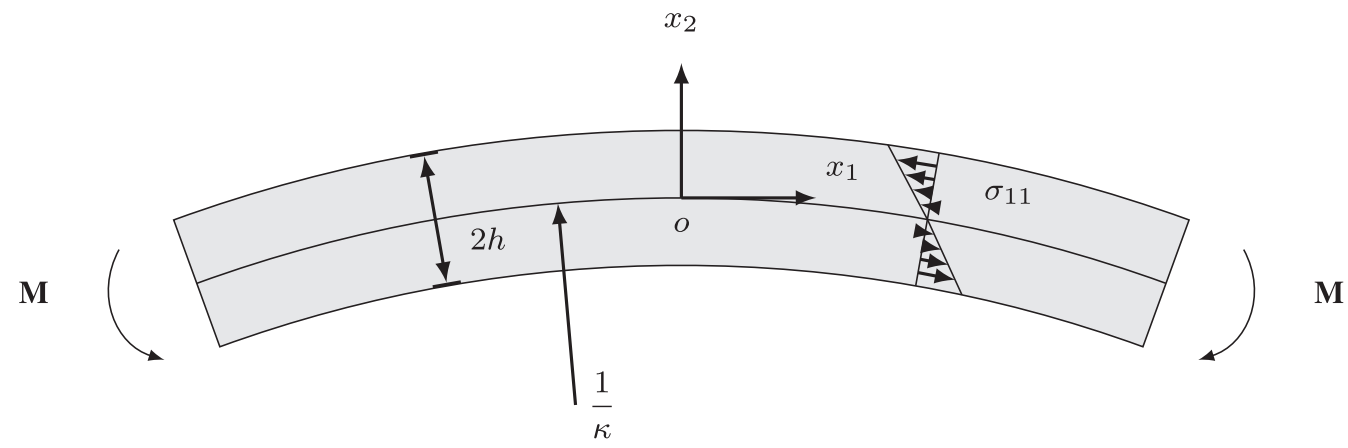

Figure 3. Schematic of a thin beam under pure bending. 
the strain displacement relations $\epsilon_{11}=u_{1,1}$ and $\epsilon_{22}=u_{2,2}$, the displacement field turns out to be,

$$
u_{1}=\kappa x_{1} x_{2}, \quad u_{2}=-\kappa \frac{x_{1}^{2}+x_{2}^{2}}{2}
$$

The deviatoric components of the stress tensor follows from Eq. (28) as,

$$
\begin{aligned}
\sigma_{11}^{\prime} & =\frac{2 \sigma_{e}}{3 \epsilon_{e}} \epsilon_{11}, \quad \sigma_{22}^{\prime}=\frac{2 \sigma_{e}}{3 \epsilon_{e}} \epsilon_{22}=-\sigma_{11}^{\prime} \\
\text { while } \epsilon_{e} & =\sqrt{\frac{4}{3}} \kappa x_{2} .
\end{aligned}
$$

Noting that $\sigma_{22}=0$ for a thin beam,

$$
\begin{aligned}
& \sigma_{22}=\sigma_{22}^{\prime}+p=0=-\sigma_{11}^{\prime}+p \text { so, } \\
& \sigma_{11}=2 \sigma_{11}^{\prime} .
\end{aligned}
$$

The moment $M$ at an arbitrary section of the beam can now be obtained using (31) and (32) as,

$$
M=\int_{-h}^{h} \sigma_{11} x_{2} b d x_{2}=2 \int_{0}^{h} \sigma_{11}^{\prime} x_{2} b d x_{2}=\left(\frac{4}{3}\right)^{\frac{n+1}{2}} \frac{\sigma_{0} b h^{2}}{(n+2)}\left(\frac{\kappa h}{\epsilon_{0}}\right)^{n} .
$$

The above moment curvature relation may be cast in a non-dimensional form as,

$$
\bar{M}=\frac{M}{\sigma_{0} b h^{2}}=\frac{1}{n+2}\left(\frac{4}{3}\right)^{\frac{n+1}{2}}\left(\frac{\kappa h}{\epsilon_{0}}\right)^{n} .
$$

Plots of $\bar{M}$ against the surface strain $\kappa h$ is contrasted in figure 4 with data from microbending experiments on thin Ni foils by Stölken \& Evans (1998). We have used $\epsilon_{0}=0.0077$ and $n=$ 0.43 to approximately fit the data for $2 h=50 \mu \mathrm{m}$. However, the variation of $\bar{M}$ versus $\kappa h$ for other values of $2 h$ clearly shows a size effect not captured by Eq. (36).

\subsection{Torsion of a thin wire}

As the next example consider the torsion of a thin wire as shown in figure 5 . The axis of the wire is along $x_{3}$ and the cross-section lies on $x_{1}-x_{2}$ plane. The wire has a radius of $a$. For a wire under torsion, the boundary conditions are

$$
\begin{aligned}
& u_{1}=u_{2}=u_{3}=0, \text { at } x_{3}=0 ; \\
& u_{1}=-\theta L x_{2}, u_{2}=\theta L x_{1}, u_{3}=0, \text { at } x_{3}=L,
\end{aligned}
$$

where $L$ is the length of the wire and $\theta$ is the twist per unit length. The only non-zero components of the strain are $\epsilon_{13}$ and $\epsilon_{23}$ and all other components $\epsilon_{11}=\epsilon_{22}=\epsilon_{33}=\epsilon_{12}=0$. The displacement field, consistent with this state of strain and (38) can be obtained as,

$$
u_{1}=-\theta x_{2} x_{3}, \quad u_{2}=\theta x_{1} x_{3}, \quad u_{3}=0 .
$$




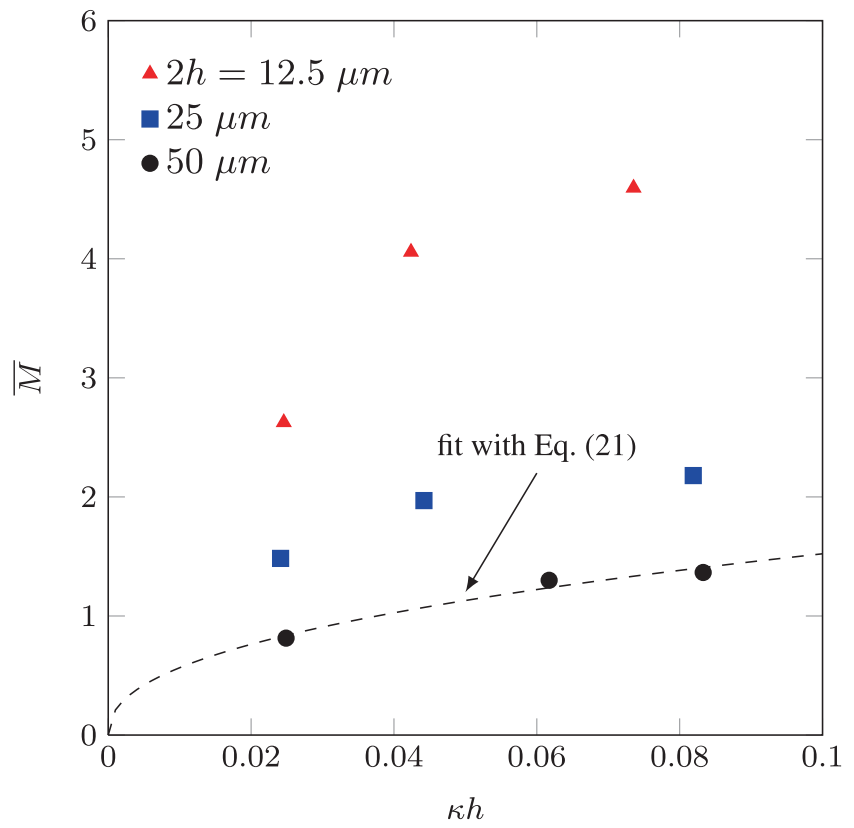

Figure 4. Results of Stölken \& Evans (1998) for microbend tests of thin Ni foils.

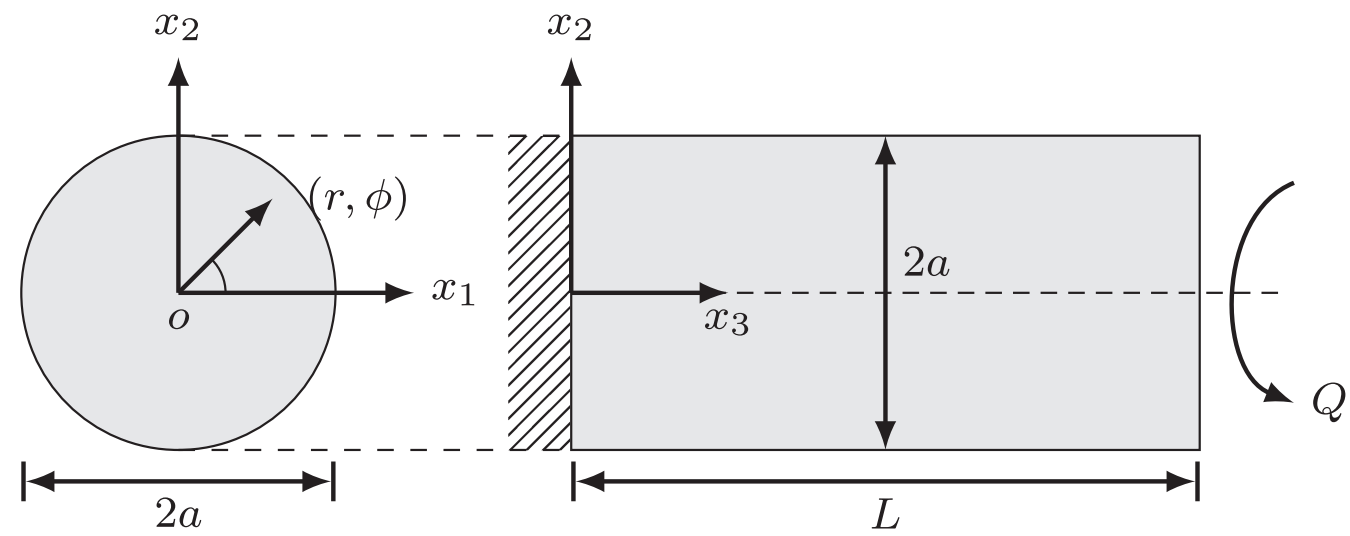

Figure 5. Geometry for torsion.

Accordingly the strain components are,

$$
\begin{aligned}
\epsilon_{13} & =\epsilon_{31}=-\frac{1}{2} \theta x_{2}, \epsilon_{23}=\epsilon_{32}=\frac{1}{2} \theta x_{1} \text { and } \\
\text { consequently, } \epsilon_{e} & =\frac{\theta}{\sqrt{3}} r .
\end{aligned}
$$

The components of the stresses follow from (28) as,

$$
\sigma_{13}=\frac{2 \sigma_{e}}{3 \epsilon_{e}} \epsilon_{13} \text { and } \sigma_{23}=\frac{2 \sigma_{e}}{3 \epsilon_{e}} \epsilon_{23}
$$


Finally the external torque $Q$ balances the moment due to the stresses acting over the crosssection of the wire:

$$
Q=\int_{0}^{2 \pi} \int_{0}^{a}\left(\sigma_{23} x_{1}-\sigma_{13} x_{2}\right) r d \phi d r=\frac{2 \pi \sigma_{0} a^{3}}{\sqrt{3}^{n+1}(n+3)}\left(\frac{\theta a}{\epsilon_{0}}\right)^{n} .
$$

Again, both the torque and the twist can be suitably normalized to yield a scale-free relation:

$$
\bar{Q}=\frac{Q}{\sigma_{0} a^{3}}=\frac{2 \pi}{\sqrt{3}^{n+1}(n+3)}\left(\frac{\theta a}{\epsilon_{0}}\right)^{n} .
$$

Similar to the bending problem, experimental observations on torsion of very thin $\mathrm{Cu}$ wires (12 to $170 \mu \mathrm{m}$ in diameter, Fleck et al 1994) reveal a similar picture of smaller being stronger. Results of their torsion tests are superposed in figure 6 where the thinnest wire $(12 \mu \mathrm{m})$ is seen to be almost 3 times stronger than the thickest ones.

\subsection{Growth of a cylindrical void under remote hydrostatic tension}

The elasto-plastic growth of a cylindrical or spherical void under hydrostatic stress is an important problem that arises in studies of ductile fracture (McClintock 1968; Rice \& Tracey 1969) and indentation (Johnson 1970). In figure 7 a schematic of cylindrical void of initial(final) radius $A(a)$, growing under a remote hydrostatic tension $\sigma_{\infty}$ is presented. Assuming axisymmetry and

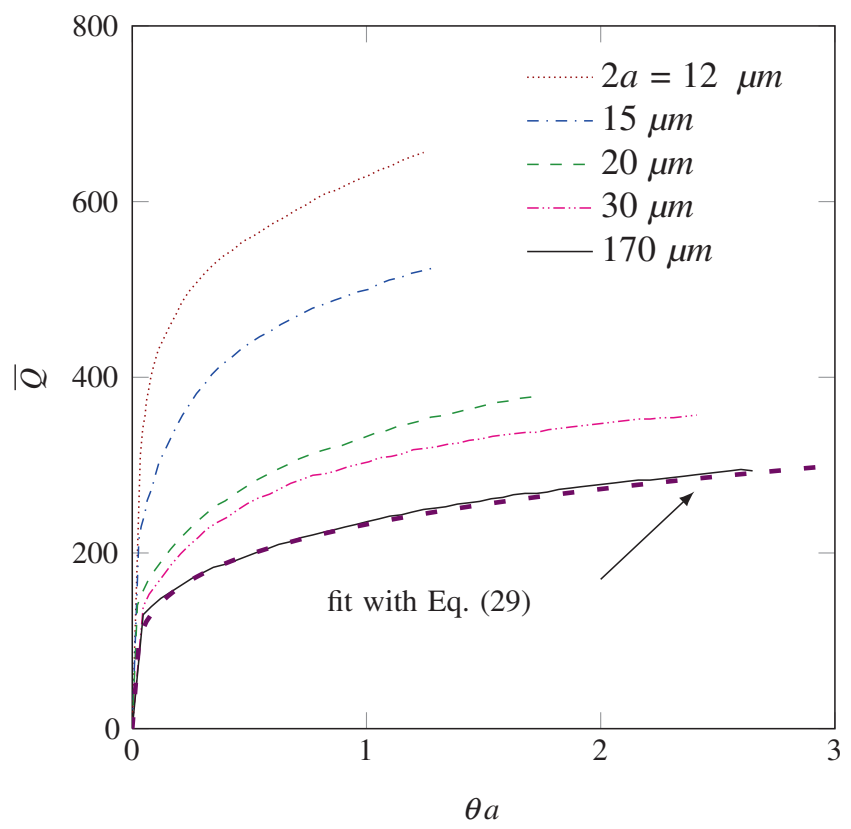

Figure 6. Results of Fleck et al (1994) for torsion tests of thin $\mathrm{Cu}$ wires. 


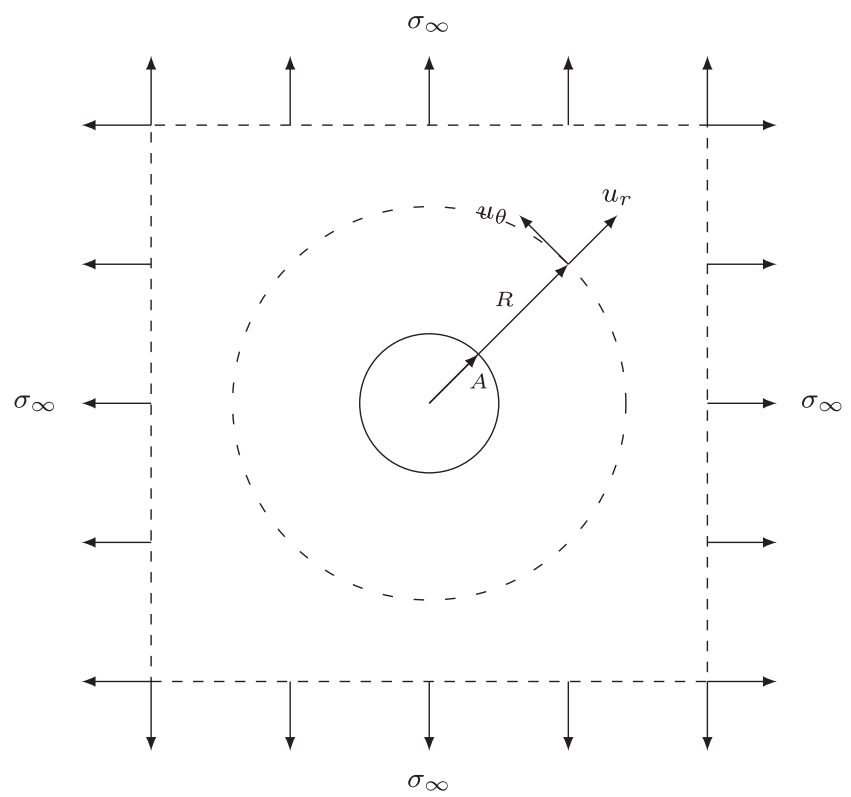

(a) Undeformed configuration

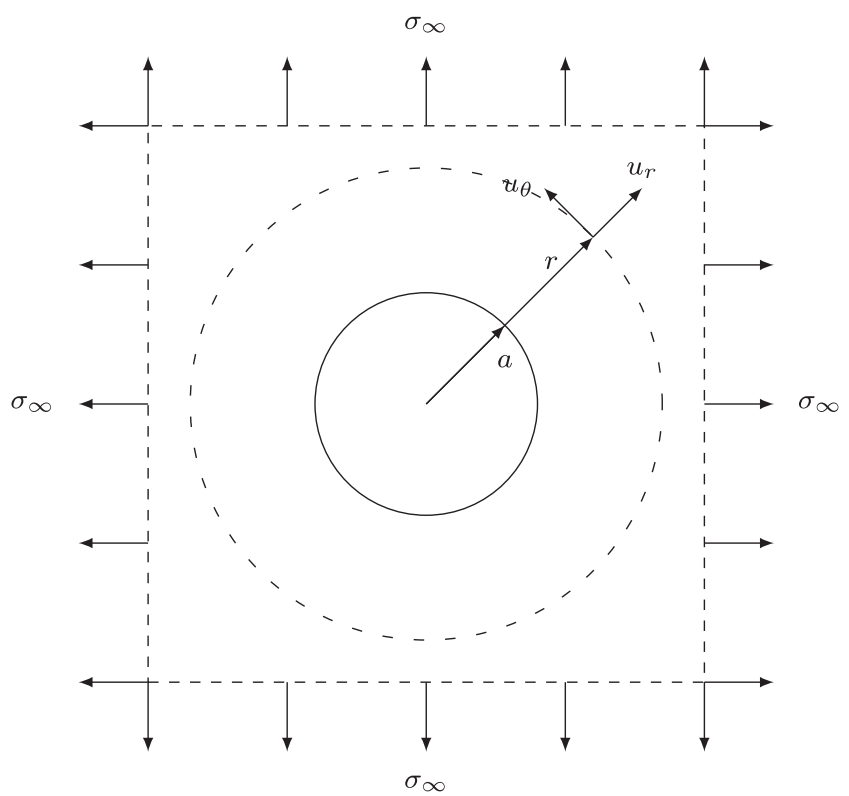

(b)

Deformed configuration

Figure 7. Growth of a cylindrical void under remote hydrostatic tension.

plane strain the deformed coordinates in terms of the undeformed coordinates $R, \Theta$ and $Z$ are given by,

$$
\begin{aligned}
& r=f(R) R, \\
& \theta=\Theta, \text { and } \\
& z=Z,
\end{aligned}
$$


where $f(R)$ is to be determined. The above motion leads to a deformation gradient (see, Eq. 8) of the form:

$$
\boldsymbol{F}=\left[\begin{array}{ccc}
f(R)+R f^{\prime} & 0 & 0 \\
0 & f(R) & 0 \\
0 & 0 & 1
\end{array}\right]
$$

The condition for incompressibility demands $\operatorname{det} \boldsymbol{F}=1$ and thus, leads to the differential equation

$$
f\left(f+R f^{\prime}\right)=1 .
$$

Solving Eq. (49) yields,

$$
\begin{aligned}
f(R) & =\left(1-\frac{A^{2}-a^{2}}{R^{2}}\right)^{1 / 2} \\
& \approx\left(1-\frac{A^{2}-a^{2}}{2 R^{2}}\right)
\end{aligned}
$$

where $A$ and $a(t)$ correspond to the radius of the void in the reference and in the deformed configurations respectively. The displacement field can now be easily determined in terms of the change in volume of the void, $\Delta V=\pi\left(A^{2}-a^{2}\right)$ as,

$$
\begin{aligned}
& u_{r}=r-R=-\frac{\Delta V}{2 \pi R}, \text { and } \\
& u_{\theta}=u_{z}=0 .
\end{aligned}
$$

For small strains, the distinction between $(R, \Theta, Z)$ and $(r, \theta, z)$ vanishes and the components of the small strain tensor then follow as,

$$
\begin{aligned}
\epsilon_{r r} & =\frac{\partial u_{r}}{\partial r}=\frac{\Delta V}{2 \pi r^{2}}, \\
\epsilon_{\theta \theta} & =\frac{1}{r} \frac{\partial u_{\theta}}{\partial \theta}+\frac{u_{r}}{r}=-\frac{\Delta V}{2 \pi r^{2}}=-\epsilon_{r r}, \text { and } \\
\epsilon_{z z} & =0 .
\end{aligned}
$$

Also, Eq. (54) yields

$$
\epsilon_{e}=\frac{2}{\sqrt{3}} \epsilon_{r r} .
$$

Using the constitutive equations in Eq. (28) the deviatoric part of the stress components is obtained as,

$$
\begin{aligned}
\sigma_{r r}^{\prime} & =\beta \epsilon_{r r}, \quad \sigma_{\theta \theta}^{\prime}=\beta \epsilon_{\theta \theta}=-\beta \epsilon_{r r}, \text { and } \sigma_{z z}^{\prime}=0, \text { where } \\
2 \beta & =\frac{4 \sigma_{0} \epsilon_{e}^{n-1}}{3 \epsilon_{0}^{n}}=\frac{\sigma_{0}}{\epsilon_{0}^{n}}\left(\frac{2}{\sqrt{3}}\right)^{n+1} \epsilon_{r r}^{n-1} .
\end{aligned}
$$

Also note that,

$$
\sigma_{r r}-\sigma_{\theta \theta}=\sigma_{r r}^{\prime}-\sigma_{\theta \theta}^{\prime}=2 \beta \epsilon_{r r} .
$$

In order to obtain a relationship between the remote hydrostatic tension $\sigma_{\infty}$ and the change of the volume $\Delta V$ of the void, the equilibrium equation needs to be integrated. Replacing $\left(\sigma_{r r}-\sigma_{\theta \theta}\right)$ and $\epsilon_{r r}$ in the equilibrium equation,

$$
\frac{\partial \sigma_{r r}}{\partial r}+\frac{\sigma_{r r}-\sigma_{\theta \theta}}{r}=0
$$


we have

$$
\int_{0}^{\sigma_{\infty}} \frac{d \sigma_{r r}}{\sigma_{0}}=-B\left(\frac{\Delta V}{2 \pi \epsilon_{0}}\right)^{n} \int_{a}^{\infty} \frac{d r}{r^{2 n+1}}
$$

which gives,

$$
\begin{aligned}
\frac{\sigma_{\infty}}{\sigma_{0}} & =-\frac{B}{2 n}\left(\frac{\Delta V}{V \epsilon_{0}}\right)^{n}, \text { where } \\
B & =\left(\frac{2}{\sqrt{3}}\right)^{n+1}, \text { and } V=2 \pi a^{2} .
\end{aligned}
$$

The solution for $\sigma_{\infty} / \sigma_{0}$ expectedly depends on the non-dimensional quantity $\Delta V / V$ through a power law relation and does not include any length scale.

\section{Size effects, geometrically necessary dislocations and strain gradients}

We have seen in section 2 that gradients of strain render a constitutive equation non-local and naturally introduce a length scale. In this section, we explore how strain gradients are related to GNDs in continuum plasticity. Plastic deformation in crystalline material is a result of dislocation motion. Dislocations on a certain slip plane start to move in a crystallographic direction when critical resolved shear stress (CRSS) is reached on that plane and in that direction. More obstacles the dislocations encounter, more stress is required to make them move continuously. In a single crystal or a polycrystalline material the strain hardening or work hardening that is observed during plastic deformation is a result of complex interactions among dislocations. During plastic deformation of single crystals of most fcc metals, dislocation slips, cross slips, entanglement and multiplications reflect as the typical three-stage flow curve (Deiter 1976). For polycrystalline materials and alloys, in addition to these factors, grain boundaries and second phase materials also serve as obstacles to dislocation motion, leading to hardening. In general, the resolved shear stress $\tau$ for a single crystal is related to the dislocation density $\rho$ according to,

$$
\tau=\tau_{0}+\alpha G b \rho^{\frac{1}{2}}
$$

where $G$ is the shear modulus, $b$ is the magnitude of Burgers vector. Further, $\tau_{0}$ is the friction stress required to move the dislocation in the absence of other dislocations arising out of the presence of solutes.

Equation (61) above shows that more stress is required for a continued flow to occur as the dislocation density increases. It is evident that the dislocation density has a direct role to play in hardening. This equation can be derived by considering that a dislocation line pinned between two hard obstacles will tend to bow out on its slip plane under the action of the resolved shear stress acting on it. The curvature of this dislocation line is related to the curvature $R$ of the bow as $\tau \sim G b / R$. For hard obstacles that are difficult to break through, $R \sim L$, the average spacing between the obstacles. Moreover, if the obstacles are jogs arising out of interaction between dislocations, the average spacing $L \simeq \rho^{-1 / 2}$. Further, assuming that the stress required to initiate slip at a potential source in a grain can result from stress concentration in a neighbouring grain ${ }^{1}$,

\footnotetext{
${ }^{1}$ The stress at the head of a pile-up (analogous to stresses at the tip of a penny shaped crack in fracture mechanics) scales as $\sqrt{D / r}$, where $r$ the distance of the source in the neighbouring grain from the tip (see, Courtney for a detailed explanation).
} 
which, in turn, may result from a pile up of dislocations, we find that $\rho \sim D^{-1}$, where $D$ is the average grain size. This leads to the well-known Hall-Petch relation.

The physical basis of strain gradient plasticity theories are rooted in the concept of geometrically necessary dislocations (GNDs), first introduced by Nye (1953), and furthered by Ashby (1970) and Ekkehart Kröner (1959). Whenever non-uniform plastic deformation is imposed on a material, an extra storage of dislocations is required to accommodate the lattice curvature (Nix $\&$ Gao 1998). On the other hand, a homogeneous state of plastic strain requires randomly generated and oriented dislocations whose overall effect on scales typical of continuum plasticity is to cause homogeneous states of deformation and stress. These dislocations are commonly called statistically stored dislocations (SSD). In other words, SSDs move and glide along slip planes in order to relax the stress while GNDs align themselves through some co-operative behavior to accommodate lattice curvatures.

\subsection{Simple examples of GND accumulation}

Before embarking on a rigorous derivation of the connection between the density of GNDs and gradients of strain, a few simple illustrations may be helpful. The need for GNDs may be demonstrated by the following simple example (Ashby 1970). Consider a square block subjected to uniform shear. It is assumed that only one slip system is active and the slip planes are parallel to $x_{1}$ direction. When the block is free to deform, the dislocation loops will reach the surface and the deformed shape will take the form as shown in figure 8b. If there is an obstacle such as a rigid particle as shown in figure $8 \mathrm{c}$, the part of the dislocation loop moving to the right (left) will be stuck at the interface while the part moving left (right) will reach the surface. Thus an additional dislocation array will exist at the boundary between the obstacle and the matrix as shown in figure $8 \mathrm{c}$.

To calculate the number of dislocations that accumulate at the boundary, it can be noticed that these are the same dislocations which would have caused an amount of slip $n b$ if they were free to move. Thus,

$$
n=\frac{\gamma l}{b}
$$

where $\gamma$ is the shear strain and $l$ is the characteristic size of the particle. Also the same number of dislocations will be accumulated at both the sides of the embedded hard particles leading to a total GNDs $n=2 \gamma l / b$.

The above number of dislocations $n$ is associated with a single embedded particle. If the number of the particles per unit volume is $N_{V}$, the total number of dislocations per unit volume will be given by,

$$
n_{T}=n N_{V}=\frac{2 N_{V} \gamma l}{b}=\frac{2 f \gamma}{b l^{2}}
$$

where $N_{V}=f / l^{3}$ and $f$ being the volume fraction of the cube shaped particle. The length of these dislocations is approximately $4 l$ for cubic particles and accordingly the density of GNDs is given by,

$$
\rho_{g}=\frac{8 f \gamma}{b l}
$$

Additionally, it can be shown by simple geometric constructions, that for a beam under bending and deforming by slip of dislocations on a single slip system (as shown in figure 9) a density of GNDs

$$
\rho_{g}=\frac{\kappa}{b}
$$




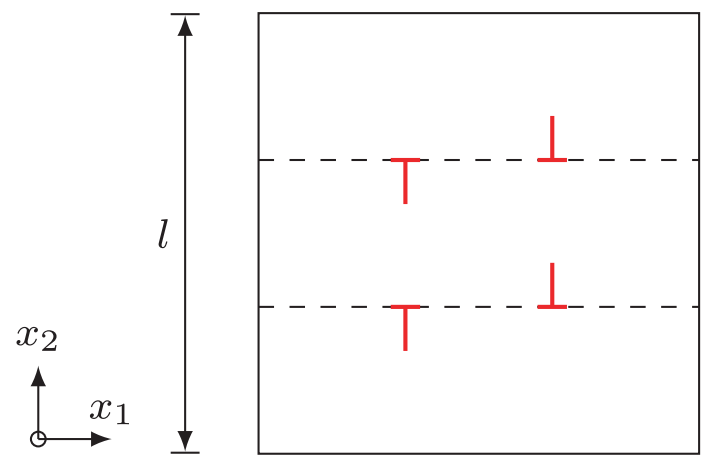

(a)

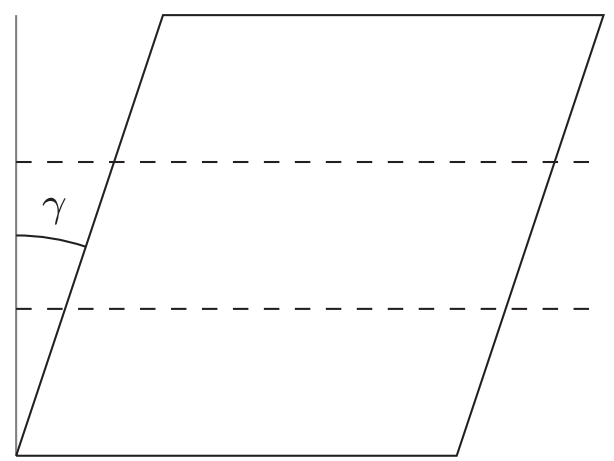

(b)

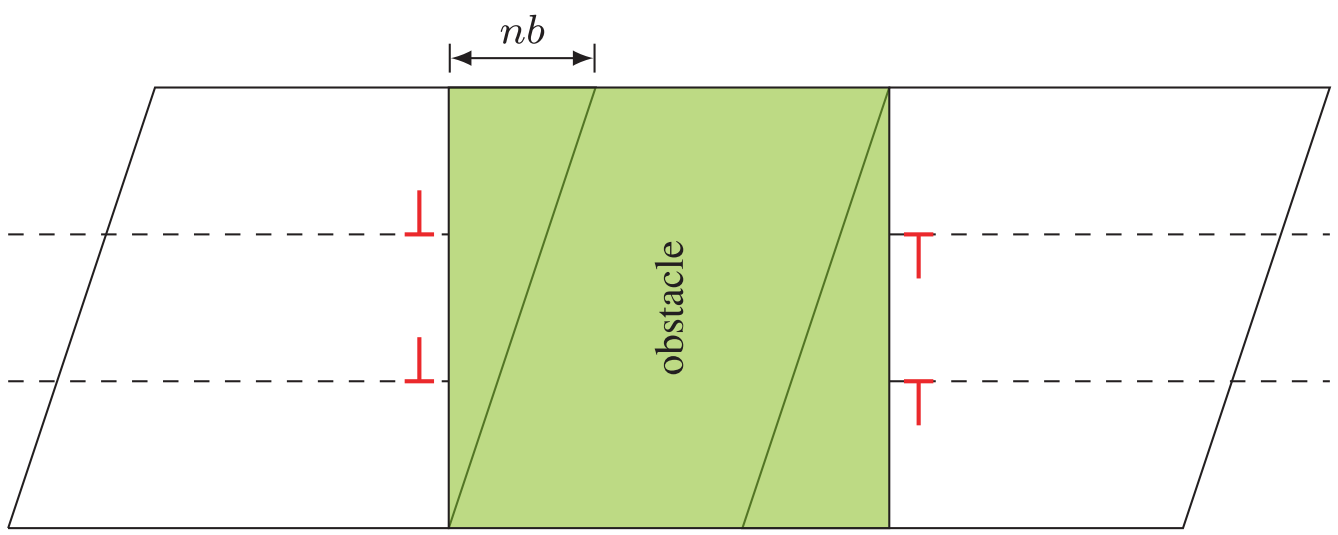

(c)

Figure 8. Schematic representation of generation of GNDs.

is required to accommodate the lattice curvature produced by the deformation (Fleck et al 1994). Similarly, the volume displaced by a conical indenter would require storage of GNDs to accommodate the shape change (Nix \& Gao 1998), leading to a density

$$
\rho_{g}=\frac{3}{2 b h} \tan ^{2} \theta
$$

Here, $\kappa$ is the curvature of the beam, $\theta$ is the angle made by the indenter with the substrate, $h$ is the depth of indentation and $b$ is the Burgers vector. These simple examples underline the fact that non-uniform strain states have to necessarily lead to accumulation of GNDs.

\subsection{Dislocation tensor: A continuum based approach for measure of dislocation density}

In this section we try to connect, within the framework of continuum single crystal plasticity, the density of GNDs to the gradient of strain. Our development will closely follow Cermelli \& Gurtin (2001), though several other authors have attempted to establish this connection (Acharya \& Bassani 2000; Nye 1953; Fleck et al 1994; Eshelby 1956; Bilby et al 1955). 


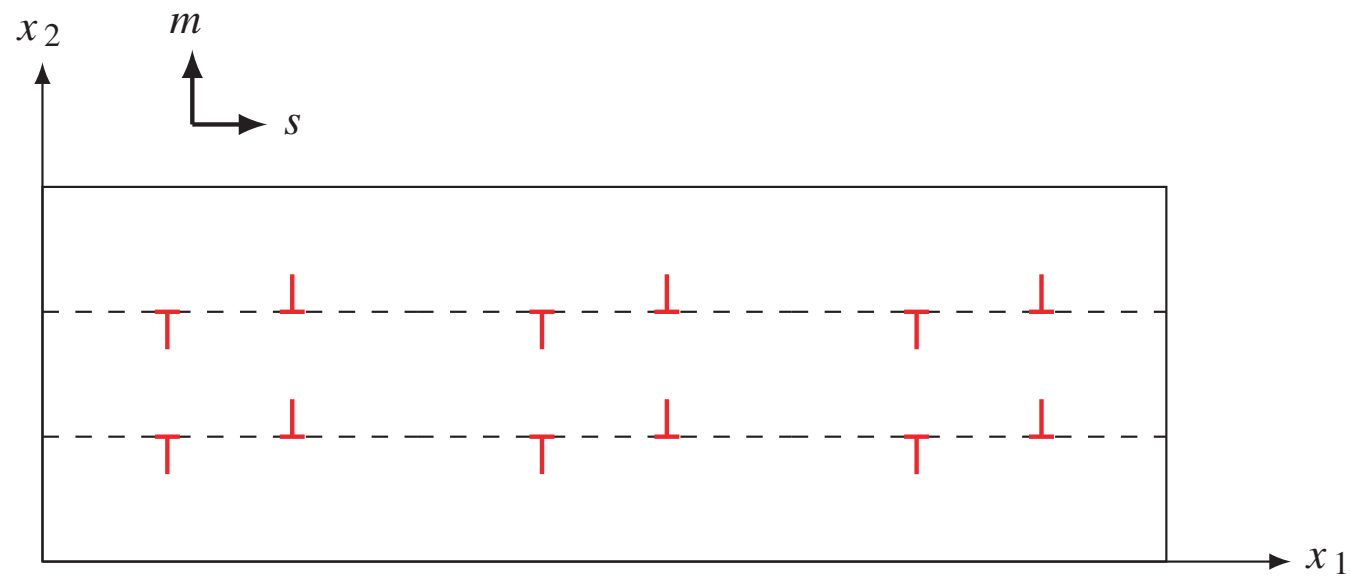

(a)

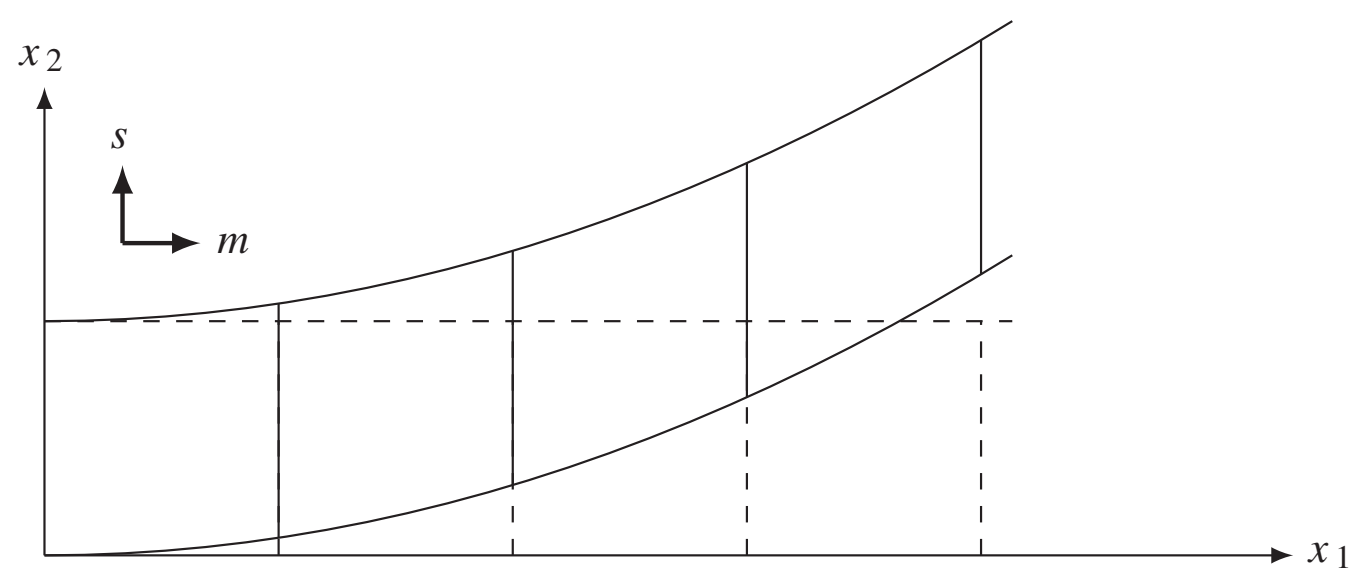

(b)

Figure 9. Schematic of slip system in a beam, where slip plane normal $\boldsymbol{m}$ and slip direction $\boldsymbol{s}$ is parallel to $x_{2}$ and $x_{1}$ respectively in (a) and to $x_{1}$ and $x_{2}$ respectively in (b).

4.2a Single crystal material: In case of a single crystal material, plastic deformation is an outcome of cumulative slips of dislocations on the preferred slip planes and in preferred directions which are tied to the crystal structure of the material. A slip system in a single crystal is defined by the slip plane, which is characterized by its unit normal $m$ and the slip direction $s$ on this plane. If multiple slip systems are active then $\left(s^{\alpha}, m^{\alpha}\right)$ denotes the $\alpha$-th slip system. Note that $s^{\alpha} \cdot m^{\alpha}=0$. If the rate of slip in the corresponding slip system is given by $\dot{\gamma}_{\alpha}^{p}$ then (Asaro \& Lubarda 2006),

$$
\boldsymbol{L}^{p}=\sum \dot{\gamma}_{\alpha}^{p} \boldsymbol{s}^{\alpha} \otimes m^{\alpha}
$$

A measure for Burgers vector has been proposed by Cermelli \& Gurtin (2001) by introducing a Burgers circuit in the reference configuration which is close in spirit to the idea proposed by Nye (1953). The Burgers vector in the intermediate configuration, determined from a Burgers 
circuit made along the boundary $\left(\partial S_{R}\right)$ of a smooth surface $S_{R}$ in the reference configuration is obtained as (see figure 2),

$$
\overline{\boldsymbol{b}}=\int_{\partial S_{R}} d \overline{\boldsymbol{x}}=\int_{\partial S_{R}} \boldsymbol{F}^{p} d \boldsymbol{X} .
$$

Note that, following Nye (1953),

$$
\overline{\boldsymbol{b}}=\int_{\partial S_{R}} \boldsymbol{\alpha} \cdot \overline{\boldsymbol{n}} d \overline{\boldsymbol{x}}
$$

where $\alpha$ is the Nye's dislocation density tensor. Note here that we are dealing with dislocations generated due to $\boldsymbol{F}^{p}$. Thus $\overline{\boldsymbol{b}}$ pertains purely to GNDs. By applying Stoke's theorem the above equation becomes,

$$
\overline{\boldsymbol{b}}=\int_{S_{R}}\left(\operatorname{Curl} \boldsymbol{F}^{p}\right)^{T} \boldsymbol{N} d A=\int_{S_{R}} \frac{1}{J^{p}}\left(\operatorname{Curl} \boldsymbol{F}^{p}\right)^{T} \boldsymbol{F}^{p T} \overline{\boldsymbol{n}} d a,
$$

where $\boldsymbol{N} d A$ and $\overline{\boldsymbol{n}} d a$ are surface elements in the reference and intermediate configuration respectively connected through the Nanson's relation (Gurtin 2010).

Note that $\bar{b}$ is non-zero only when the deformation is not compatible. A dislocation tensor $\boldsymbol{G}$ may be defined as,

$$
\boldsymbol{G}=\frac{1}{J^{p}} \boldsymbol{F}^{p}\left(\operatorname{Curl} \boldsymbol{F}^{p}\right)
$$

so that

$$
\overline{\boldsymbol{b}}=\int_{S_{R}} \boldsymbol{G}^{T} \overline{\boldsymbol{n}} d a .
$$

Consequently $\boldsymbol{G}^{T} \overline{\boldsymbol{n}}$ gives a measure of Burgers vector per unit area of the plane $\overline{\boldsymbol{n}}$ in the intermediate configuration. For plane strain case, Cermelli \& Gurtin (2001) has shown that the dislocation tensor simplifies to,

$$
G=e \otimes g,
$$

where $\boldsymbol{e} \equiv \boldsymbol{e}_{3}$ and $\boldsymbol{g}=\left(\operatorname{Curl} \boldsymbol{F}^{p}\right)^{T} \boldsymbol{e} / J^{p}$. In this case, the local Burgers vector per unit area becomes $\boldsymbol{G}^{T} \boldsymbol{e}=\boldsymbol{g}^{T}$. The connection between $\boldsymbol{g}$ and $\mathrm{Curl}^{p}$ shows that the density of GNDs is indeed related to the gradients of the plastic deformation. We can now illustrate the above result with a simple example.

4.2b Simple case with one slip plane: Consider the problem of shear in a single crystal beam as shown in figure 9(a). The beam is experiencing shear by slip of dislocation dipoles on a single slip plane with unit normal $\boldsymbol{m}$, in a direction $\boldsymbol{s}$. For simplicity, $\boldsymbol{m}$ and $\boldsymbol{s}$ are assumed to be parallel to $x_{2}$ and $x_{1}$ direction respectively. For such a slip system, Eq. (67) gives the only non-zero component of $\boldsymbol{L}^{p}$ as $L_{12}=\dot{\gamma}\left(x_{1}\right)$, where $\dot{\gamma}\left(x_{1}\right)$ is only a function of $x_{1}$ but not of time. In order to solve for $\boldsymbol{F}^{p}$ for this problem Eq. (13) 3 is employed, which produces the following set of differential equations,

$$
\begin{aligned}
\dot{F}_{11}^{p} & =\dot{\gamma} F_{21}^{p}, \quad \dot{F}_{12}^{p}=\dot{\gamma} F_{22}^{p}, \quad \dot{F}_{13}^{p}=\dot{\gamma} F_{23}^{p} \text { and } \\
\dot{F}_{i j}^{p} & =0, \quad \forall \text { other } i, j .
\end{aligned}
$$


If it is assumed that at time $t=0$, the body occupies the reference configuration, then $\left.\boldsymbol{F}^{p}\right|_{t=0}=$ I. Using this condition, the solution to the Eq. (74) becomes,

$$
\boldsymbol{F}^{p}=\left[\begin{array}{lll}
1 & \gamma & 0 \\
0 & 1 & 0 \\
0 & 0 & 1
\end{array}\right] .
$$

Note that $J^{p}=1$. Thus $\boldsymbol{A}=\operatorname{Curl} \boldsymbol{F}^{p 2}$ is calculated as,

$$
\left[\begin{array}{ccc}
-\frac{\partial \gamma}{\partial X_{3}} & 0 & 0 \\
0 & 0 & 0 \\
\frac{\partial \gamma}{\partial X_{1}} & 0 & 0
\end{array}\right]
$$

The measure of local Burgers vector per unit area $g$ turns out to be,

$$
\boldsymbol{g}=\left\{\begin{array}{c}
\frac{\partial \gamma}{\partial X_{1}} \\
0 \\
0
\end{array}\right\}=\frac{\partial \gamma}{\partial X_{1}} e_{1}
$$

It can readily be seen that the density of dislocations associated with $g$ then becomes,

$$
\rho_{g}=\frac{|\boldsymbol{g}|}{b}=\frac{1}{b} \frac{\partial \gamma}{\partial X_{1}},
$$

which shows the connection between the density of GNDs and the strain gradient. A phenomenological route produces the same result to the above problem, as illustrated in Fleck et al (1994). Using the same approach for a different slip system shown in figure $9 \mathrm{~b}$ it can be shown that the local Burgers vector per unit area becomes zero requiring no dislocation storage during the deformation.

${ }^{2}$ By definition, curl of a vector $v$ is

$$
\operatorname{Curl} v=e_{i j k} v_{j, i} \boldsymbol{e}_{k},
$$

where $e_{i j k}$ is the permutation tensor. Consider a constant vector $\boldsymbol{c}$ and a second order tensor $\boldsymbol{T}$ such that,

$$
\nabla \times(\boldsymbol{c} \cdot \boldsymbol{T})=e_{i j k} c_{m} T_{m j, i} \boldsymbol{e}_{k}=\left(e_{i j k} T_{m j, i} \boldsymbol{e}_{k} \boldsymbol{e}_{m}\right) \cdot \boldsymbol{c},
$$

so that

$$
\operatorname{Curl} \boldsymbol{T}=e_{i j k} \frac{\partial T_{m j}}{\partial X_{i}} \boldsymbol{e}_{k} \boldsymbol{e}_{m}
$$

A useful identity involving curl of a second order tensor is 


\section{Towards a thermodynamically consistent gradient plasticity theory}

In the previous section, the density of GNDs was connected to $\nabla_{X} \times \boldsymbol{F}^{p}$. For polycrystals, (Aifantis 1984; Fleck \& Hutchinson 2001), the internal power is expressed as,

$$
W_{I}=\int_{\Omega}\left[\sigma: D^{e}+\tau^{p} \dot{\epsilon}^{p}+\xi^{p} \cdot \nabla \dot{\epsilon}^{p}\right] d v
$$

Here, the effective plastic strain rate $\dot{\epsilon}^{p}=\sqrt{2 \boldsymbol{D}^{p}: \boldsymbol{D}^{p} / 3}$ and its gradient $\nabla \dot{\epsilon}^{p}$ are used as measures of plastic strain and strain gradient respectively. The existence of an effective plastic stress $\tau^{p}$ and a higher order stress $\xi^{p}$ conjugate of $\dot{\epsilon}^{p}$ and $\nabla \dot{\epsilon}^{p}$ respectively also needs to be assumed. More elaborate theories (e.g. Gurtin \& Anand 2009) use other measures like $\nabla \dot{\boldsymbol{E}}^{p}$ and a higher order stress $\boldsymbol{K}^{p}$, which is a third order tensor. However, here we stick to the simpler formulation where gradients of $\dot{\epsilon}^{p}$, a scalar measure of strain represents the effect of the GNDs.

We mention in passing here that, the quantity $\operatorname{Curl} \boldsymbol{F}^{p}$ whose components may be written as

$$
F_{k m}^{p}=e_{k j i} \frac{\partial^{2} \bar{x}_{m}}{\partial X_{i} \partial X_{j}},
$$

where $\overline{\boldsymbol{x}}$ denotes the intermediate configuration. This tensor (see, Fleck 1994; Fleck \& Hutchinson 2001) represents the gradient of the rotation vector associated with the motion from reference to the intermediate configuration. As shown in Eq. (28) of Fleck \& Hutchinson (2001), it is possible to relate the gradient of rotation to the gradients of the equivalent plastic strain $\epsilon^{p}$ but the connections are complicated. Here, we take the gradient of the equivalent plastic strain rate as the embodiment of all gradient effects arising out of GNDs as a purely phenomenological fact.

Each component in the above expression quantifies the work done by various agencies during deformation. The first term on the right is the internal power required to deform the body elastically. The effective stress quantity $\tau^{p}$ has been termed as a microstress (Gurtin \& Anand 2009; Gudmundson 2004) in view of the fact that the second term embodies the power needed to push dislocations and make them flow through the microstructure. The last term is the power needed to generate the GNDs necessary to accommodate the deformation.

Assume that a scalar field $e$ represents the internal energy per unit deformed volume. Further, heat flow is described by a vector heat flux $\boldsymbol{q}$ and a scalar heat generation term $r$. The rate form of the first law of thermodynamics states that the rate of generation of internal energy $U$ equals the internal power and the rate of net heat flow, giving

$$
U=W+Q,
$$

so that,

$$
\overline{\int_{\Omega} e d v}=\int_{\Omega}\left[\boldsymbol{\sigma}: \boldsymbol{D}^{e}+\left(\tau^{p} \dot{\epsilon}^{p}+\boldsymbol{\xi}^{p} \cdot \nabla \dot{\epsilon}^{p}\right)\right] d v-\int_{\partial \Omega} \boldsymbol{q} \cdot \boldsymbol{n} d s+\int_{\Omega} r d v .
$$

Note that $\dot{\overline{(~)}}$ denotes a total time derivative. The above form can be localized in a standard way (Holzapfel 2000) yielding:

$$
\dot{e}=\sigma: \boldsymbol{D}^{e}+\left(\tau^{p} \dot{\epsilon}^{p}+\xi^{p} \cdot \nabla \dot{\epsilon}^{p}\right)-\nabla \cdot \boldsymbol{q}+r,
$$


as the local energy balance applicable all points in $\Omega$. The Clausius-Planck inequality that embodies the second law of thermodynamics can be phrased as

$$
\boldsymbol{\sigma}: \boldsymbol{D}^{e}+\tau^{p} \dot{\epsilon}^{p}+\boldsymbol{\xi}^{p} \cdot \nabla \dot{\epsilon}^{p}-\dot{\psi}-\dot{T} \eta \geq 0
$$

Another crucial assumption involves the constitutive relation for $\psi$. In a significant departure from conventional plasticity, it is assumed that,

$$
\psi=\psi^{e}\left(\boldsymbol{E}^{e}\right)+\psi^{p}\left(\epsilon^{p}, \nabla \epsilon^{p}\right),
$$

where the elastic part $\psi^{e}$ may be assumed to be of the standard quadratic, isotropic form,

$$
\psi^{e}=\mu \boldsymbol{E}^{e}: \boldsymbol{E}^{e}+\frac{1}{2} \lambda\left(\operatorname{tr} \boldsymbol{E}^{e}\right)^{2}
$$

The plastic part $\psi^{p}$ depends on both $\epsilon^{p}$ and $\nabla \epsilon^{p}$. When Eq. (84) is inserted into the ClausiusPlanck inequality Eq. (83), we obtain the dissipation condition:

$$
\left(\sigma-\boldsymbol{F}^{e} \frac{\partial \psi^{e}}{\partial \boldsymbol{E}^{e}} \boldsymbol{F}^{e T}\right): \boldsymbol{D}^{e}+\left(\tau^{p}-\frac{\partial \psi^{p}}{\partial \epsilon^{p}}\right) \dot{\epsilon}^{p}+\left(\xi^{p}-\frac{\partial \psi^{p}}{\partial \nabla \epsilon^{p}}\right) \cdot \nabla \dot{\epsilon}^{p}-\dot{T} \eta \geq 0 .
$$

Following the usual argument that the second law of thermodynamics must be satisfied under all conceivable processes, at a given point $\boldsymbol{x}$ and time $t$, it is possible to prescribe arbitrary values of $\boldsymbol{D}^{e}$ such that the inequality is violated. This necessarily implies that in the inequality, the first term within brackets, none of whose terms depend on the elastic stretch rate, must vanish. This argument leads to the constitutive equation for $\sigma$ as

$$
\boldsymbol{\sigma}=\boldsymbol{F}^{e} \frac{\partial \psi^{e}}{\partial \boldsymbol{E}^{e}} \boldsymbol{F}^{e T}
$$

Additionally, if isothermal conditions with $\dot{T}=0$ are assumed to prevail, the dissipation inequality reads,

$$
\bar{\tau}^{p} \dot{\epsilon}^{p}+\bar{\xi}^{p} \cdot \nabla \dot{\epsilon}^{p} \geq 0
$$

where

$$
\begin{aligned}
& \bar{\tau}^{p}=\tau^{p}-\frac{\partial \psi^{p}}{\partial \epsilon^{p}}, \text { and } \\
& \overline{\boldsymbol{\xi}}^{p}=\boldsymbol{\xi}^{p}-\frac{\partial \psi^{p}}{\partial \nabla \epsilon^{p}},
\end{aligned}
$$

are the dissipative parts of the microstress $\tau^{p}$ and the higher order stress $\xi^{p}$.

\subsection{Variational principle}

The expression of internal virtual power follows from Eq. (79) as

$$
\delta W_{I}=\int_{\Omega}\left(\sigma: \delta \boldsymbol{D}^{e}+\tau^{p} \delta \dot{\epsilon}^{p}+\boldsymbol{\xi}^{p} \cdot \delta \nabla \dot{\epsilon}^{p}\right) d v .
$$


Divergence theorem can be applied to the last terms in the above equation to yield,

$$
\int_{\Omega} \xi^{p} \cdot \delta \nabla \dot{\epsilon}^{p} d v=\int_{\partial \Omega} \xi^{p} \cdot \boldsymbol{n} \delta \dot{\epsilon}^{p} d s-\int_{\Omega} \nabla \cdot \xi^{p} \delta \dot{\epsilon}^{p} d v
$$

Guided by the term $\left(\boldsymbol{\xi}^{p} \cdot \boldsymbol{n}\right) \delta \dot{\epsilon}^{p}$, we assume that power is expended by a microscopic traction $\chi(\boldsymbol{n})$ conjugate to $\dot{\epsilon}^{p}$ acting on the boundary $\partial \Omega$ of the deformed configuration. This microscopic traction acts in addition to the usual traction $T_{i}=\sigma_{i j} n_{j}$ and is an outcome of the constitutive dependence on $\nabla \dot{\epsilon}^{p}$. We will discuss the consequences of the "higher order traction" term in section 6 . Thus the external virtual power has the form,

$$
\delta W_{E}=\int_{\partial \Omega}\left(\boldsymbol{T} \cdot \delta \dot{\boldsymbol{u}}+\chi \delta \dot{\epsilon}^{p}\right) d s+\int_{\Omega} \boldsymbol{b} \cdot \delta \dot{\boldsymbol{u}} d v
$$

In indicial notation, the principle of virtual power becomes

$$
\int_{\Omega}\left(\sigma_{i j} \delta D_{i j}^{e}+\tau^{p} \delta \dot{\epsilon}^{p}+\xi_{i}^{p} \delta \dot{\epsilon}_{, i}^{p}\right) d v=\int_{\Omega} b_{i} \delta \dot{u}_{i} d v+\int_{\partial \Omega}\left(T_{i} \delta \dot{u}_{i}+\chi \delta \dot{\epsilon}^{p}\right) d s .
$$

At this stage, we restrict ourselves to situations where $\boldsymbol{F}^{e} \approx \boldsymbol{I}$ and therefore,

$$
\boldsymbol{D}=\boldsymbol{D}^{e}+\boldsymbol{D}^{p}
$$

is valid. Moreover, codirectionality of $\boldsymbol{D}^{p} /\left|\boldsymbol{D}^{p}\right|$ and $\boldsymbol{\sigma}^{\prime} /\left|\boldsymbol{\sigma}^{\prime}\right|$ is also assumed, such that

$$
\frac{\boldsymbol{\sigma}^{\prime}}{\left|\boldsymbol{\sigma}^{\prime}\right|}=\frac{\boldsymbol{D}^{p}}{\left|\boldsymbol{D}^{p}\right|}=\boldsymbol{N}^{p}
$$

where $\boldsymbol{N}^{p}$ is the flow direction (Gurtin et al 2010). The above restriction on plastic flow is commonly imposed in Mises' plasticity. Defining $\sigma_{e}=\sqrt{\frac{3}{2} \sigma^{\prime}: \sigma^{\prime}}$, the above constraint yields

$$
\boldsymbol{D}^{p}=\frac{3}{2} \dot{\epsilon}^{p} \frac{\boldsymbol{\sigma}^{\prime}}{\sigma_{e}}
$$

Thus,

$$
\boldsymbol{\sigma}: \delta \boldsymbol{D}^{e}=\boldsymbol{\sigma}: \delta \boldsymbol{D}-\boldsymbol{\sigma}^{\prime}: \delta \boldsymbol{D}^{p}=\boldsymbol{\sigma}: \delta \nabla \dot{\boldsymbol{u}}-\sigma_{e} \delta \dot{\epsilon}^{p} .
$$

The last term follows from the fact that $\dot{\epsilon}^{p} \delta \dot{\epsilon}^{p}=\frac{2}{3} \boldsymbol{D}^{p}: \delta \boldsymbol{D}^{p}$. Finally, the principle for virtual power $\delta W_{I}=\delta W_{E}$ can be written as,

$$
\int_{\Omega}\left[\sigma: \delta \nabla \dot{\boldsymbol{u}}+\left(\tau^{p}-\sigma_{e}\right) \delta \dot{\epsilon}^{p}+\boldsymbol{\xi}^{p} \cdot \delta \nabla \dot{\epsilon}^{p}\right] d v=\int_{\Omega} \boldsymbol{b} \cdot \delta \dot{\boldsymbol{u}} d v+\int_{\partial \Omega}\left(\boldsymbol{T} \cdot \delta \dot{\boldsymbol{u}}+\chi \delta \dot{\epsilon}^{p}\right) d s .
$$

The divergence theorem can be applied to the first and third terms on the left giving,

$$
\begin{gathered}
-\int_{\Omega}\left[\sigma_{i j, j} \delta \dot{u}_{i}+\left(\tau^{p}-\sigma_{e}\right) \delta \dot{\epsilon}^{p}-\xi_{i, i} \delta \dot{\epsilon}^{p}\right] d v+\int_{\partial \Omega}\left(\sigma_{i j} n_{j} \delta \dot{u}_{i}+\xi_{i} n_{i} \delta \dot{\epsilon}^{p}\right) d s \\
=\int_{\Omega} b_{i} \delta \dot{u}_{i} d v+\int_{\partial \Omega}\left(T_{i} \delta \dot{u}_{i}+\chi \delta \dot{\epsilon}^{p}\right) d s .
\end{gathered}
$$


Arguing that the above must hold for any arbitrary volume element $\Omega$ and any values of the virtual quantities $\delta \dot{u}_{i}$ and $\delta \dot{\epsilon}^{p}$, we obtain the usual macroscopic equation of motion,

$$
\sigma_{i j, j}+b_{i}=0 \text { in } \Omega \text {. }
$$

Additionally, we get the microscopic balance equation,

$$
\tau^{p}=\sigma_{e}+\xi_{i, i}^{p} \text { in } \Omega
$$

On the surface $\partial \Omega$, we must satisfy the macro-traction relation,

$$
T_{i}=\sigma_{i j} n_{j}
$$

as well as the micro-traction relation (or the higher order boundary condition),

$$
\chi=\xi_{i}^{p} n_{i}
$$

The microforce balance equation physically implies that the net resistance to plastic flow is composed of $\sigma_{e}$, which is required to drive SSDs through the microstructure, and $\xi_{i, i}$ required to create GNDs necessary for causing curvature of the underlying lattice. Note that $\xi^{p}$ has units of moment per unit area.

\subsection{Thermodynamically consistent framework}

We can now go back to the inferences drawn from the dissipation inequality to derive a thermodynamically consistent constitutive framework. Note that the microforce balance requires that

$$
\sigma_{e}=\bar{\tau}^{p}+\frac{\partial \psi^{p}}{\partial \epsilon^{p}}-\left(\bar{\xi}_{i}^{p}+\frac{\partial \psi^{p}}{\partial \epsilon_{, i}^{p}}\right)_{, i} .
$$

As an example, if we assume that $\psi^{p}=\frac{1}{2} \beta\left|\nabla \epsilon^{p}\right|^{2}$ (with $\beta>0$ ), and the higher order stress is completely energetic, i.e. $\bar{\xi}^{p}=\mathbf{0}$, we get,

$$
\frac{\partial \psi^{p}}{\partial \nabla \epsilon^{p}}=\beta \nabla \epsilon^{p}, \text { and } \frac{\partial \psi^{p}}{\partial \epsilon^{p}}=0
$$

This implies that $\tau^{p}=\bar{\tau}^{p}$ or that $\tau^{p}$ is completely dissipative, while the higher order microscopic stress is energetic. These lead to,

$$
\sigma_{e}=\bar{\tau}^{p}-\beta \frac{\partial^{2} \epsilon^{p}}{\partial x_{k} \partial x_{k}}
$$

The constitutive equation for the dissipative microstress may be taken as,

$$
\bar{\tau}^{p}=Y\left(\epsilon^{p}\right) .
$$

The above equation explicitly states that the stress required to cause the SSDs to flow depends only on the accumulated plastic strain $\epsilon^{p}$. In that sense, Eq. (107) resembles a yield condition. 
Under these assumptions the above framework reduces to that proposed by Aifantis (1984), namely,

$$
\sigma_{e}=Y\left(\epsilon^{p}\right)-\beta \nabla^{2} \epsilon^{p}
$$

\subsection{Fleck-Hutchinson's constitutive relations as a special case}

An alternate formulation due to Fleck \& Hutchinson (2001), uses a rate dependent gradient plasticity formulation (Borg et al 2006) where a generalized effective strain rate

$$
\dot{E}^{p 2}=\dot{\epsilon}^{p 2}+l_{*}^{2} \dot{\epsilon}_{, i}^{p} \dot{\epsilon}_{, i}^{p},
$$

is defined where $l_{*}$ is a material length parameter. The internal virtual power can be written in the framework of Eq. (91). They also define an effective stress $\sigma_{c}$ such that,

$$
\tau^{p} \delta \dot{\epsilon}^{p}+\xi^{p} \cdot \delta \nabla \dot{\epsilon}^{p}=\sigma_{c} \delta \dot{E}^{p},
$$

with

$$
\begin{aligned}
\tau^{p} & =\frac{\sigma_{c} \dot{\epsilon}^{p}}{\dot{E}^{p}} \text { and } \\
\xi^{p} & =\frac{l_{*}^{2} \sigma_{c}}{\dot{E}^{p}} \nabla \dot{\epsilon}^{p} .
\end{aligned}
$$

Thus,

$$
\sigma_{c}^{2}=\tau^{p 2}+\frac{1}{l_{*}^{2}} \xi_{i}^{p} \xi_{i}^{p}
$$

The internal virtual power in Eq. (91) becomes,

$$
\delta W_{I}=\int_{\Omega}\left(\sigma: \delta \boldsymbol{D}^{e}+\sigma_{c} \delta \dot{E}^{p}\right) d v .
$$

In this formulation, owing to the choice of the effective strain rate, $\sigma_{c}$ takes the role of $\sigma_{e}$ in section 5.1. The microforce balance expectedly yields Eq. (102). Assuming that $\tau^{p}$ and $\xi^{p}$ are completely dissipative, (i.e. $\bar{\tau}^{p}=\tau^{p}, \bar{\xi}=\boldsymbol{\xi}$ ) it is easy to see that the rate dependent formulation satisfies the dissipation inequality Eq. (88) identically. Interestingly, a completely dissipative higher order stress is admissible in the rate dependent formulation.

Note that the assumption of completely dissipative $\tau^{p}$ and $\xi^{p}$ are different in spirit from the model due to Aifantis (1984) (Eq. (106)), where we assumed $\xi^{p}$ to be completely energetic. A completely energetic $\xi^{p}$ implies that the $\nabla \dot{\epsilon}^{p}$ can be reversed on reversing the higher order stress $\xi^{p}$.

In rate independent formulations due to Fleck \& Hutchinson (2001), the dissipation inequality in Eq. (88) poses a severe problem as pointed out by Gudmundson (2004) and Gurtin \& Anand (2009). In such cases, $\dot{\epsilon}^{p}>0$ may be assumed for all cases, and,

$$
\psi^{p}=\psi^{p}\left(\epsilon^{p}(t), \epsilon_{, k}^{p}(t)\right)
$$


Thus, the dissipation inequality can be written as,

$$
\left[\tau^{p}(t)-\frac{\partial \psi^{p}\left(\epsilon^{p}, \epsilon_{, k}^{p}\right)}{\partial \epsilon^{p}}\right] \dot{\epsilon}^{p}+\left[\xi_{k}^{p}(t)-\frac{\partial \psi^{p}\left(\epsilon^{p}, \epsilon_{, k}^{p}\right)}{\partial \epsilon_{, k}^{p}}\right] \dot{\epsilon}_{, k}^{p} \geq 0 .
$$

A subtle consideration is used here to prove that $\bar{\xi}_{k}^{p}=\xi^{p}-\frac{\partial \psi^{p}}{\partial \epsilon_{, k}^{p}}$, has to vanish if the above equation is never violated. In a problem in plasticity $\dot{\epsilon}^{p}$ is greater than 0 and continuous. However, the same is not applicable to $\dot{\epsilon}_{, k}^{p}$, which can be piecewise continuous and varied independently. So, while the first term within the square brackets in the above equation is not independent of $\dot{\epsilon}^{p}$, the term inside the second square bracket can potentially be independent of $\dot{\epsilon}_{, k}^{p}$. Thus, a $\dot{\epsilon}_{, k}^{p}$ may be prescribed that violates Eq. (116). To prevent this eventuality,

$$
\bar{\xi}_{k}^{p}=\xi_{k}^{p}-\frac{\partial \psi^{p}}{\partial \epsilon_{, k}^{p}}=0,
$$

implying that $\xi^{p}$ has to be completely energetic in a rate independent formulation. It is worthwhile to remind the reader that this restriction does not apply to a rate dependent formulation as shown in Eq. (111). It should be noted here that earlier rate independent strain gradient theories due to Fleck \& Hutchinson (2001) have recently been shown to violate the Clausius-Planck inequality (Gurtin \& Anand 2009) for certain strain paths. This is demonstrated for a simplified form of the rate independent model in Appendix A.

A rate independent model for strain gradient plasticity that is consistent with the ClausiusPlanck inequality has recently been proposed by Hutchinson (2012). Here they have introduced a free energy of the form

$$
\psi^{p}\left(\epsilon^{p}, \epsilon_{k}^{p}\right)=U^{p}\left(E^{p}\right)-U^{p}\left(\epsilon^{p}\right) .
$$

By definition,

$$
\begin{aligned}
U^{p}\left(E^{p}\right) & =\int_{0}^{E^{p}} \sigma_{c} d E^{p}, \text { and } \\
U^{p}\left(\epsilon^{p}\right) & =\int_{0}^{\epsilon^{p}} \sigma_{c} d \epsilon^{p} .
\end{aligned}
$$

Thus $\psi^{p}$ is the free energy resulting from an energetic difference in driving both GNDs and SSDs $\left(U^{p}\left(E^{p}\right)\right)$ and SSDs $\left(U^{p}\left(\epsilon^{p}\right)\right)$ only. The free energy defined here vanishes in the absence of strain gradients and models the recoverable energy due to storage of GNDs. Noting that

$$
\begin{aligned}
& \frac{\partial U^{p}\left(E^{p}\right)}{\partial \epsilon^{p}}=\frac{\partial U^{p}}{\partial E^{p}} \frac{\partial E^{p}}{\partial \epsilon^{p}}=\frac{\sigma_{c} \epsilon^{p}}{E^{p}}, \text { and } \\
& \frac{\partial U^{p}\left(\epsilon^{p}\right)}{\partial \epsilon^{p}}=\sigma_{c},
\end{aligned}
$$

we have,

$$
\bar{\tau}^{p}=\sigma_{c},
$$


while the energetic part of $\tau^{p}$ is $\frac{\sigma_{c}}{E^{p}} \epsilon^{p}-\sigma_{c}$. Similarly,

$$
\frac{\partial \psi^{p}}{\partial \epsilon_{, k}^{p}}=l_{*}^{2} \frac{\sigma_{c}}{E^{p}} \epsilon_{, k}^{p}
$$

gives the energetic part of $\underline{\xi}^{p}$ while, as proved earlier for rate independent theories, to satisfy the dissipation inequality, $\xi^{p}=\mathbf{0}$. With this rate independent formulation, for a case of plastic loading with $\dot{\epsilon}^{p}>0$, the dissipation inequality is always satisfied.

\subsection{Simple problems in gradient plasticity}

We conclude the discussion with rederiving the solutions to the problems addressed in section 3 using a gradient plasticity theory. We wish to illustrate that introduction of a length scale in such a theory can indeed produce a size effect. As it was already established that a gradient plasticity theory with a rate-dependent constitutive relationship of the form given in Eq. (112) can guarantee positive plastic dissipation, such a constitutive model is used for the following analyses along with an assumption of negligible elastic deformation. The uniaxial stress-strain response is assumed to follow a rate-dependent model as,

$$
\sigma_{c}=\sigma_{0}\left(1+\frac{E^{p}}{\epsilon_{0}}\right)^{n}\left(\frac{\dot{E}^{p}}{\dot{\epsilon}_{0}}\right)^{m}=g\left(\frac{\dot{E}^{p}}{\dot{\epsilon}_{0}}\right)^{m},
$$

where $m$ is the strain-rate sensitivity parameter.

5.4a Bending of thin beams: The problem of bending, as described in Section 3.3, is once again addressed here. The displacements, stress and strain components are defined in Eq. (2932). Neglecting elastic strains in Eq. (79) the internal power for the problem becomes,

$$
M \dot{\kappa}=2 b \int_{0}^{h}\left(\tau^{p} \dot{\epsilon}^{p}+\xi^{p} \cdot \nabla \dot{\epsilon}^{p}\right) d x_{2}=\frac{2 b}{\dot{\epsilon}_{0}^{n}} \int_{0}^{h} g \dot{E}^{p^{m+1}} d x_{2} .
$$

The terms $M$ and $\kappa$ are resultant moment and rotation that have been applied on the beam.

Similarly, under negligible elastic deformation $\epsilon_{e}=\epsilon^{p}=\sqrt{\frac{4}{3}} \kappa x_{2}$. So, Eq. (110) gives,

$$
\dot{E}^{p}=\sqrt{\frac{4}{3}} \dot{\kappa}\left(x_{2}^{2}+l_{*}^{2}\right)^{\frac{1}{2}},
$$

and the above integral reduces to,

$$
M=2 b g\left(\frac{\dot{\kappa}}{\dot{\epsilon}_{0}}\right)^{m}\left(\frac{4}{3}\right)^{\frac{m+1}{2}} \int_{0}^{h}\left(x_{2}^{2}+l_{*}^{2}\right)^{\frac{m+1}{2}} d x_{2} .
$$

In deriving the above spatial variation of $g$ has been ignored. It is evident from Eq. (127) that the moment under this framework is directly related to the domain size through the ratio $l_{*} / h$. It can also be noticed that for $l_{*} \ll h$ the result reduces to conventional predictions. 
5.4b Torsion of a thin wire: The torsion problem defined in section 3.4 is also solved in a gradient plasticity framework. By a set of arguments similar to those given in the previous section and making use of the strain components defined in Eq. (41), we arrive at the following variational form for the torque,

$$
Q \dot{\theta}=\frac{2 \pi}{\dot{\epsilon}_{0}^{m}} \int_{0}^{a} g \dot{E}^{p^{m+1}} r d r .
$$

Noting that,

$$
\dot{E}^{p}=\frac{\dot{\theta}}{\sqrt{3}}\left(r^{2}+l_{*}^{2}\right)^{\frac{1}{2}},
$$

for the torsion problem the above integration results in the following form for the torque,

$$
\begin{aligned}
\frac{Q}{a^{3}} & =\frac{2 \pi g}{m+3}\left(\frac{\dot{\theta} a}{\dot{\epsilon}_{0}}\right)^{m}\left[\left(1+\frac{l_{*}^{2}}{a^{2}}\right)^{\frac{m+3}{2}}-\left(\frac{l_{*}}{a}\right)^{m+3}\right] \\
& =Q_{0}\left[\left(1+\frac{l_{*}^{2}}{a^{2}}\right)^{\frac{m+3}{2}}-\left(\frac{l_{*}}{a}\right)^{m+3}\right],
\end{aligned}
$$

where $Q_{0}$ is the value of $Q / a^{3}$ for the limiting case $l_{*} / a \rightarrow 0$. The normalized torque $Q / Q_{0} a^{3}$ is plotted against $a / l_{*}$ in figure 10. It exhibits a size effect when $l_{*}$ is close to $a$, whereas the effect dies down with increasing $a / l_{*}$. The results of the torsion tests by Fleck et al (1994) as shown in figure 6 has also been fitted with this model in figure 10 for two different values of surface strain $\theta a=1$ and 0.3 with $l_{*}=9 \mu \mathrm{m}$. Assuming that $Q / a^{3}$ for $2 a=170 \mu \mathrm{m}$ in their

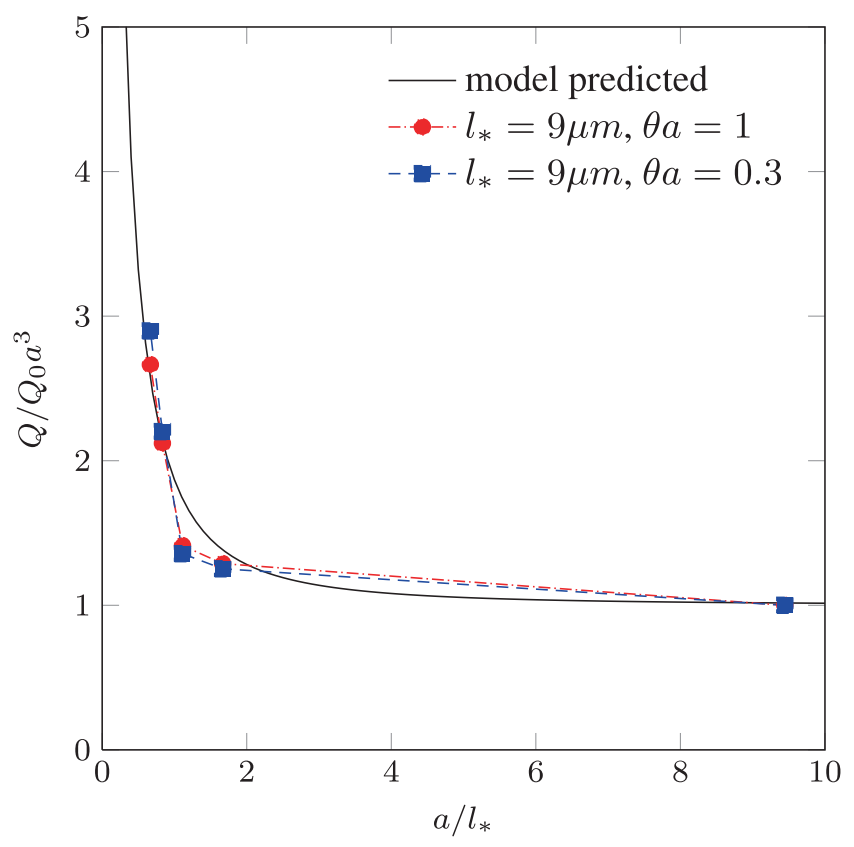

Figure 10. Variation of normalized torque with $a / l_{*}$. 
result represents the value for the bulk material it has been considered as $Q_{0}$ and the subsequent values for other samples has been normalized with $Q_{0}$. Interestingly, results corresponding to both the values of surface strains fit well with $l_{*}=9 \mu \mathrm{m}$.

\section{Higher order boundary conditions}

The first term in Eq. (93) gives the variation of the external power expended on the deformed external boundary of the body. In addition to the power due to the traction $\boldsymbol{T}$, power is expended by the higher order traction $\chi=\xi_{i} n_{i}$ (Eq. 104). A variational principle of the kind given in Eq. (91) will necessitate a prescription of either the higher order stress $\xi^{p}$ or the plastic strain $\epsilon^{p}$ on the boundary. It is worthwhile here to dwell on the physical nature of the higher order traction. It can be observed that if a constitutive relation of the form given in Eq. (141) is used then the boundary conditions can be specified in terms of $\epsilon^{p}$ and $\nabla \epsilon^{p}$. This enables one to model problems with hard interfaces or boundaries which prohibit dislocation motions. A rigid interface that is impermeable to dislocation motion across it has $\epsilon^{p}=0$. For example, consider the shear of a thin rectangular layer between two rigid platens as shown in figure 11 . The rigid platens do not allow dislocations to pass through and accordingly $\epsilon^{p}=0$ is specified at $X_{2}= \pm L / 2$. Figure 12 shows the distribution of $\epsilon^{p}$ across the layer, obtained from a finite element computations for different values of $l_{*}$. The expected distribution for conventional plasticity has also been shown. The distribution of $\epsilon^{p}$ exhibits a boundary layer, whose thickness depends on the characteristic length scale $l_{*}$ chosen. Similar results have been obtained in discrete dislocation simulations
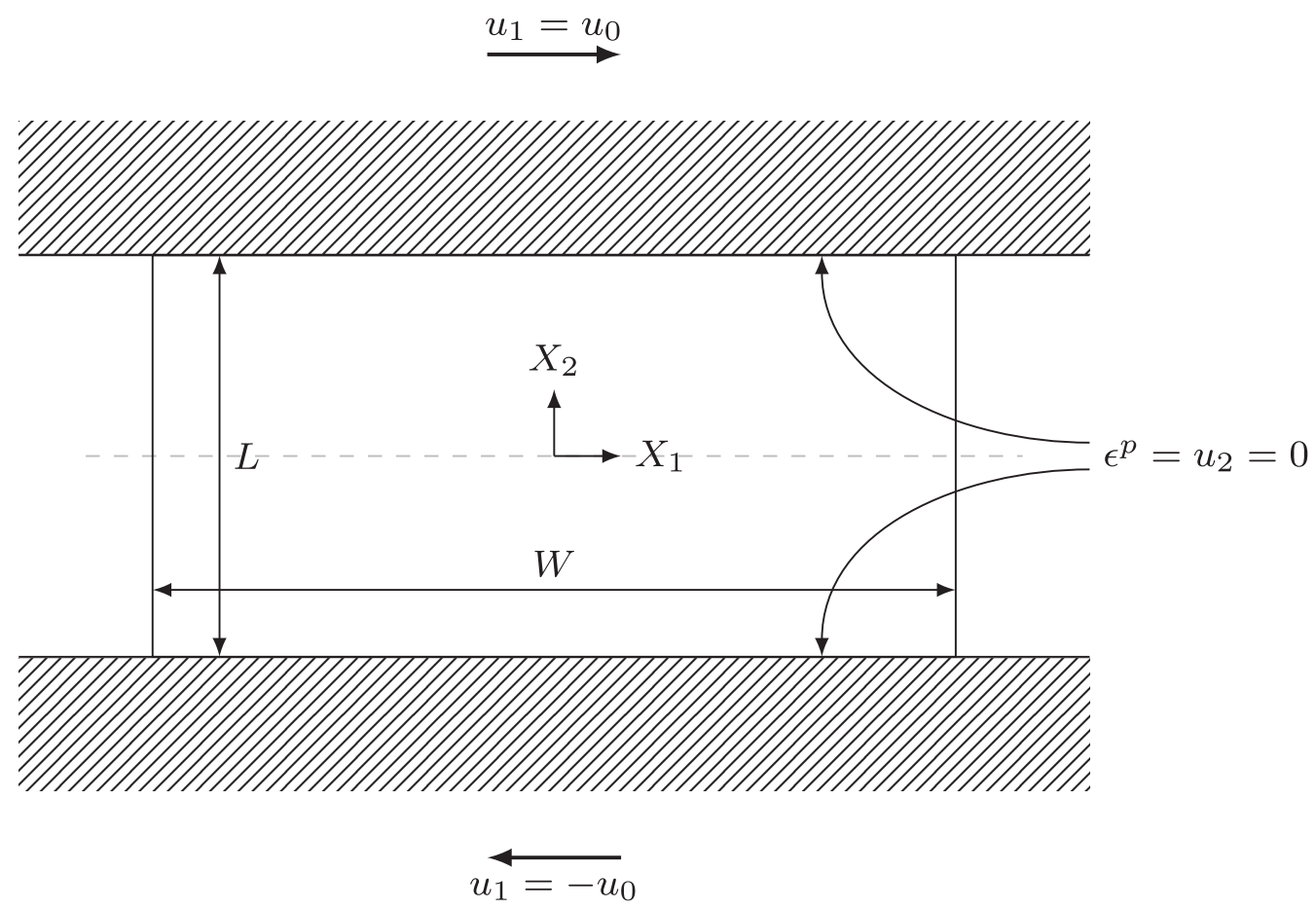

Figure 11. Schematic of a constrained shear of a thin rectangular layer between two rigid punches. 


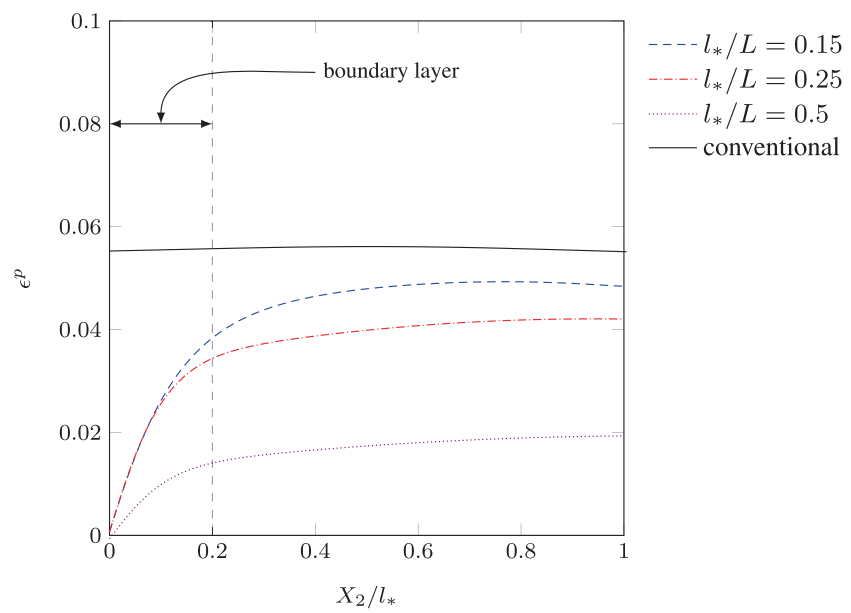

Figure 12. Distribution of $\epsilon^{p}$ across the thickness in the constrained layer.

by Shu et al (2001) and finite element simulations by Fleck \& Hutchinson (2001). On the contrary, if instead the natural boundary condition $\chi=\xi^{p} \cdot \boldsymbol{n}=\epsilon_{, 2}^{p}=0$ was used (which means dislocations pass without resistance through the boundaries, Niordson \& Hutchinson 2003), no boundary layer appears in $\epsilon^{p}$ and the conventional solution is recovered.

Apart from the extreme cases of perfectly permeable and completely impermeable boundaries, it is possible to account for surface or interface energies within this framework. Gudmundson (2004) has given a generalized prescription by considering dissipation at the interface between two materials deforming plastically. In his formulation Eq. (91) is rewritten as

$$
\delta W_{I}=\int_{\Omega}\left(\sigma: \delta \dot{\boldsymbol{D}}^{e}+\tau^{p} \delta \dot{\epsilon}^{p}+\boldsymbol{\xi}^{p} \cdot \delta \nabla \dot{\epsilon}^{p}\right) d v+\int_{\delta \Omega_{i}}\left(M^{1} \delta \dot{\epsilon}^{p 1}+M^{2} \delta \dot{\epsilon}^{p 2}\right) d s,
$$

where $\delta \Omega_{i}$ is an internal boundary separating two microstructurally distinct regions and $M^{\alpha}$ (with $\alpha=1,2$ ) are work conjugates to the plastic strain rates $\dot{\epsilon}^{p \alpha}$ at the interface. An arbitrary variation of $\epsilon^{p}$ at the boundary $\delta \Omega_{i}$ will produce the boundary conditions,

$$
\begin{aligned}
& \xi^{1} \cdot n^{1}=M^{1} \text { and, } \\
& \xi^{2} \cdot n^{2}=M^{2}
\end{aligned}
$$

at $\Omega_{i}$. The simplest prescription is to assume that no plastic dissipation occurs at the interface and consequently, the constitutive relation for $M^{1}$ and $M^{2}$ can be given in terms of the free energy of the interface $\Psi_{i}\left(\epsilon^{p 1}, \epsilon^{p 2}\right)$ as,

$$
M^{\alpha}=\frac{\partial \Psi_{i}\left(\epsilon^{p 1}, \epsilon^{p 2}\right)}{\partial \epsilon^{p \alpha}}, \text { where } \alpha=1,2 .
$$

Here, $\Psi_{i}$ is the energy density of an interface as a function of the plastic strains on either side. This formulation has been used by Aifantis \& Willis (2005) to model microcompression experiments. 
Internal boundaries between elastic and elasto-plastic parts of the body pose a subtle problem. Obviously, $\dot{\epsilon}^{p}=0$ in the elastic part. However, $\nabla \dot{\epsilon}^{p}$ need not be zero at an elastic-plastic boundary. This implies that a higher order traction acts on such a boundary. Several authors have used a vanishing higher order traction at such boundaries (Niordson \& Hutchinson 2003; Niordson 2003; Niordson \& Tvergaard 2006). For certain problems (Niordson \& Redanz 2004) it has been found that results are not significantly influenced by whether we choose $\chi=0$ or $\dot{\epsilon}^{p}=0$ at such boundaries. However, as shown in Niordson (2008), this is not generally the case. Effective techniques for dealing with elasto-plastic boundaries are still being worked on. An elegant solution to this issue lies in implementing a rate-dependent theory (Borg et al 2006) where no internal boundaries between elastic and plastic regions exist. It should also be noted that the formulation due to (Gudmundson et al 2004) discussed earlier, can handle elasto-plastic boundaries when either $\epsilon^{p 1}$ or $\epsilon^{p 2}$ is zero.

\section{Conclusions}

In this review, we started by pointing out that the nonlocal behavior can be captured by introducing a gradient term in the constitutive relationship. A length scale parameter $l_{*}$ is thus introduced which controls the size dependent behavior (see Eq. (5)). This is the basic essence of most of the strain gradient plasticity theories till date. Most early experiments (Fleck et al 1994; Stölken \& Evans 1998; Ma \& Clarke 1995; Ranjana Saha \& William D Nix 2002; McElhaney et al 1998; George M Pharr et al 2010; Poole et al 1996) have also revealed that size effects manifest only at the micron/sub-micron scales. Consequently, experimentally determined values of $l_{*}$ for various metals fall within this range. In fact, fitting the numerical results to the experimentally available data, $l_{*}$ is found to be $\approx 4 \mu \mathrm{m}$ for $\mathrm{Cu}$ (Fleck et al 1994) and $\approx 5 \mu \mathrm{m}$ for Ni (Stölken \& Evans 1998). On the other hand, experimental data for nano/micro-indentations suggest values of $l_{*}$ in the range $0.2-0.5 \mu \mathrm{m}$ for Ag, Mo and W single crystals (Matthew R Begley \& John W Hutchinson 1998). At this moment, it is difficult to accept $l_{*}$ as a material parameter. It seems to depend on the specific problem being addressed. A physical interpretation of $l_{*}$ remains elusive.

The fundamental length scales for a crystalline material which govern the plastic deformation are the Burgers vector $b$, dislocation spacing, size of the second phase particles, mean free path of a dislocation, etc. Efforts have been made to connect $l_{*}$ with the above length scales. For example, William D Nix \& Huajian Gao (1998) and Evans \& Hutchinson (2009) have shown that $l_{*}$ can be interpreted as $L^{2} / b$, where $L$ is the mean spacing between SSDs or the second phase particles or any other obstacles. However, $l_{*}$ is associated with gradient of strain or GNDs. Hence, an interpretation of $l_{*}$ in terms of length scales associated with SSDs appears to be dubious. William D Nix \& Huajian Gao (1998) model for indentation gives a measure of the length scale $h^{*}$ in indentation, in terms of the hardness $H_{0}$ or the yield strength $\sigma_{0}$ of the material in the absence of GNDs/strain gradients. This suggests that $h^{*}$ might be related to material property and should evolve with plastic deformation as $\sigma_{0}$ undergoes changes owing to the strain hardening. In fact, Evans \& Hutchinson (2009) have introduced a $l_{*}$, linearly dependent on plastic strain, to demonstrate that a better fit to the experimental results is obtained. Al-Rub \& Voyiadjis (2004) have also shown that $l_{*}$ can be given in terms of the mean dislocation spacing $L$. Additionally, using Gracio's (Gracio 1994) approximation for $L$ in terms of grain size $d$ and $\epsilon^{p}$, it is shown that,

$$
l_{*} \approx \frac{\delta d}{\delta+d \epsilon^{p^{\frac{1}{n}}}},
$$


where $\delta$ is a constant on the order of $1 \mu \mathrm{m}$. Another interpretation of $l_{*}$ has been given by Evans \& Hutchinson (2009). Here, $l_{*}$ is correlated with the average distance $\bar{d}_{g}$ that GNDs travel, through a dissipative plastic deformation. They show that the potential $U^{p}\left(E^{p}\right)$ may be expressed in terms of the densities of SSDs $\rho_{S}$ and GNDs $\rho_{g}$ as

$$
U^{p}\left(E^{p}\right) \approx \sigma_{0}\left(\rho_{s} b \bar{d}_{s}+\rho_{g} b \bar{d}_{g}\right) .
$$

Identifying $\epsilon^{p}=\rho_{s} b \bar{d}_{s}$ and $\rho_{g}=\eta / b$ (where $\eta$ is the magnitude of the gradient of $\epsilon^{p}$ ), $l_{*}$ turns out to be $\bar{d}_{g}$.

It can be recalled here that, plastic dissipation under a gradient plasticity framework is caused solely by a dissipative microstress $\bar{\tau}^{p}$, and an energetic $\xi^{p}$. As a consequence of the higher order stress $\xi^{p}$ being completely energetic, gradients of strain are supposed to be reversible. From this viewpoint, assumption of $\rho_{g}$ contributing towards dissipation according to Eq. (136) is questionable. In another interpretation, Shu et al (2001), by comparing discrete dislocation simulations and gradient plasticity simulations, have shown that $l_{*}$ correlates to the thickness of the region over which dislocations are piled up at a constrained layer.

Recent experimental methods, such as the use of EBSD (Electron Backscattering Diffraction) and Orientation Imaging Microscopy (OIM), have made it possible to estimate the density of GNDs in suitable experiments. For example, measurement of local lattice curvature in ElDasher et al (2003) show pile up of GNDs of about $30 \mu \mathrm{m}$ in thickness near the grain boundary. A systematic study of computing density of GNDs from the measurement of lattice rotation through EBSD, has been furthered by Kysar $(2007,2010)$ and Pantleon (2008). However, these experiments are limited by in-plane lattice rotation measurements only. This poses a problem in computing $\rho_{g}$, as only two components of the gradient of lattice rotations can be experimentally measured. However, this can be circumvented by assuming plane strain to prevail or computing the out of plane rotations by the use of Focused Ion Beam (FIB) milling (Kiener et al 2006). Nevertheless, the problem of computing the density $\rho_{g}$ might remain indeterminate as any combination of slip systems might produce the same deformation. This is further elaborated below for a specific situation.

An explicit connection between the curvature tensor $\chi_{i j}$ (see Eq. (21)) and the Nye's dislocation tensor $\alpha_{i j}$, in the absence of elastic strain gradients, has been achieved by Arsenlis $\&$ Parks (1999) through the following relation:

$$
\chi_{i j}=-\alpha_{j i}+\frac{1}{2} \delta_{i j} \alpha_{k k},
$$

where $\chi_{i j}$ are the components of rotational gradient tensor $\theta_{i, j}$. The above relation provides a route to compute the individual densities $\rho_{g}^{n}$ in the $n^{\text {th }}$ active slip system. To this end, $\boldsymbol{\alpha}$ is redefined for a case with $m$ slip systems as

$$
\alpha_{i j}=\sum_{n=1}^{m} \rho_{g}^{n} b_{i}^{n} t_{j}^{n},
$$

where $\boldsymbol{b}^{n}$ and $\boldsymbol{t}^{n}$ are the Burgers vector and the unit tangent vector of the dislocation line respectively (Arsenlis \& Parks 1999). As a simple case, consider a plane strain situation. Thus, only dislocations with $\boldsymbol{t}=-\boldsymbol{e}_{3}$ are responsible for slip and the only non-zero components of $\boldsymbol{\alpha}$ are $\alpha_{13}$ and $\alpha_{23}$ (note that, for plane strain only $b_{1}$ and $b_{2}$ are applicable). Also, noting that only $\chi_{31}$ 
and $\chi_{32}$ are measurable from in-plane EBSD studies, Eq. (138) and (137) can be combined to give,

$$
\begin{aligned}
& \sum_{n=1}^{m} \rho_{g}^{n} b_{1}^{n}=\chi_{31}, \quad \text { and } \\
& \sum_{n=1}^{m} \rho_{g}^{n} b_{2}^{n}=\chi_{32} .
\end{aligned}
$$

Here, $\boldsymbol{b}$ is the material property and can be found for each slip system $n$. However, it can be noticed that, in the above case the solution to $\rho_{g}^{n}$ might become indeterminate depending on the choice of slip system (here, for $m>2$, the system of equations become indeterminate). Solutions pertaining to more slip systems can be obtained, if measurements of more components of $\chi$ from experiments are made possible. Alternatively, a solution to the above system of equations still can be obtained through optimization techniques (Arsenlis \& Parks 1999; Jeffrey W Kysar et al 2007). In fact, with the assumptions made above, Jeffrey W Kysar et al (2007) have estimated the distribution of $\rho_{g}$ from the data obtained for wedge indentations of $\mathrm{Cu}$ and $\mathrm{Al}$ single crystal specimens with $m=3$. These experiments have the potential to shed light on the connection between the density of GNDs and macroscopic gradients of strain.

Finally, the size effect is associated with non-uniform deformation. However, several uniaxial compression and tensile experiments on micro/nano structured pillars (Michael D Uchic \& Dennis M Dimiduk 2005; Dimiduk et al 2005; Michael D Uchic et al 2006; Budiman et al 2008a) have shown strong size effect in apparently uniform deformation. Gradients of strains or additional dislocation density due to GNDs $\left(\rho_{g}\right)$ as such, do not play any role in these cases, towards enhancing the strength. It has been argued that the observed increase in strength due to the uniform deformation results from a rapid starvation of the structures of the small dislocation sources present in it at the outset (Julia R Greer \& William D Nix 2006). Discrete Dislocation Dynamics simulations by Tang et al (2007) seems to confirm that owing to the small size $(\approx 200 \mathrm{~nm})$ of the micro/nanopillars, dislocations escape to the surface rapidly, creating a dislocation starved region in the deforming volume. However, these experiments do not necessarily discredit a strain gradient theory of plasticity. Questions regarding strain gradients introduced by gallium ions from the focused ion beam milling process and/or loading misalignments, sample taper remain and have been discussed in the literature (Budiman et al 2008b).

\section{Acknowledgements}

The authors gratefully acknowledge the financial support from the Department of Science and Technology, Govt. of India for the present work.

\section{Appendix A}

A rate independent constitutive relation for an incremental formulation is given by Fleck \& Hutchinson (2001) where

$$
\dot{\tau}^{p}=h\left(E^{p}\right) \dot{\epsilon}^{p}, \text { and } \dot{\xi}_{i}=l_{*}^{2} h\left(E^{p}\right) \dot{\epsilon}_{, i}^{p} .
$$

Here $h\left(E^{p}\right)=d \sigma_{c} / d E^{p}$ is the hardening modulus evaluated at $E^{p}$. Replacing the constitutive relations in the microforce balance $\tau^{p}=\sigma_{e}+\xi_{i, i}^{p}$, results in a flow rule which is a second order 
differential equation in terms of $\epsilon^{p}$. The flow rule thus obtained is essentially a variant of the one given in Eq. (109):

$$
\dot{\sigma}_{e}=h \dot{\epsilon}^{p}-l_{*}^{2} h \nabla^{2} \dot{\epsilon}^{p} .
$$

In deriving the above, the spatial variation of $h$ has been ignored. However, it has been argued (Morton E Gurtin \& Lallit Anand 2009; Peter Gudmundson 2004) that the above constitutive relations are not consistent with the second law of the thermodynamics for certain strain loading paths. In fact, Morton E Gurtin \& Lallit Anand (2009) have shown that for a particular loading path the above relations fail to satisfy the dissipation inequality (Eq. (88)). A simplified proof of this fact is presented here.

Following (Morton E Gurtin \& Lallit Anand 2009) we start with a simpler form of the constitutive equations given in Eq. (141) namely,

$$
\dot{\tau}^{p}=H\left(\epsilon^{p}\right) \dot{\epsilon}^{p}, \text { and } \dot{\xi}_{k}=G\left(\epsilon^{p}\right) \dot{\epsilon}_{, k}^{p} .
$$

Incidentally, the violation of the dissipation inequality can also be proved for the constitutive model in Eq. (141) but is substantially more tedious. Additionally we assume that,

$$
\begin{aligned}
& \epsilon^{p}=\epsilon_{, k}^{p}=0, \text { at } t=0, \text { and } \\
& \dot{\epsilon}^{p} \geq 0, \text { for } t \geq 0 .
\end{aligned}
$$

By integrating the above constitutive relations, consistent with the above initial conditions one obtains,

$$
\tau^{p}=S\left(\epsilon^{p}\right) \text { and } \xi_{k}=\int_{0}^{t} G\left(\epsilon^{p}\right) \epsilon_{, k}^{p} d t
$$

with an assumption that $\tau^{p}(0)=0$ and $\xi_{k}(0)=0$. As $\bar{\xi}^{p}=\mathbf{0}$ for rate independent models, the higher order stress

$$
\xi_{k}^{p}=\frac{\partial \psi^{p}}{\partial \epsilon_{, k}^{p}}
$$

which, from Eq. (145), can be written as

$$
\xi_{k}^{p}=\left.\frac{\partial \psi^{p}\left(\epsilon^{p}, \epsilon_{, k}^{p}\right)}{\partial \epsilon_{, k}^{p}}\right|_{t}=\int_{0}^{t} G\left(\epsilon^{p}(y)\right) \dot{\epsilon}_{, k}^{p}(y) d y .
$$

Since for no strain history should we detect a possible violation of the dissipation inequality, we choose a strain history ( shown in figure 13) of the form

$$
\begin{aligned}
& \epsilon_{\delta}^{p}(y)=0, \text { for } 0 \leq y \leq t-\delta \\
& \epsilon_{\delta}^{p}(t)=\epsilon^{p}(t) .
\end{aligned}
$$

Under this history, the right side of Eq. (147) becomes

$$
\int_{0}^{t} G\left(\epsilon_{\delta}^{p}(y)\right) \dot{\epsilon}_{, k}^{p}(y) d y=\left[G(0) \epsilon_{, k}^{p}(y)\right]_{0}^{t-\delta}+\int_{t-\delta}^{t} G\left(\epsilon_{\delta}^{p}(y)\right) \dot{\epsilon}_{, k}^{p}(y) .
$$




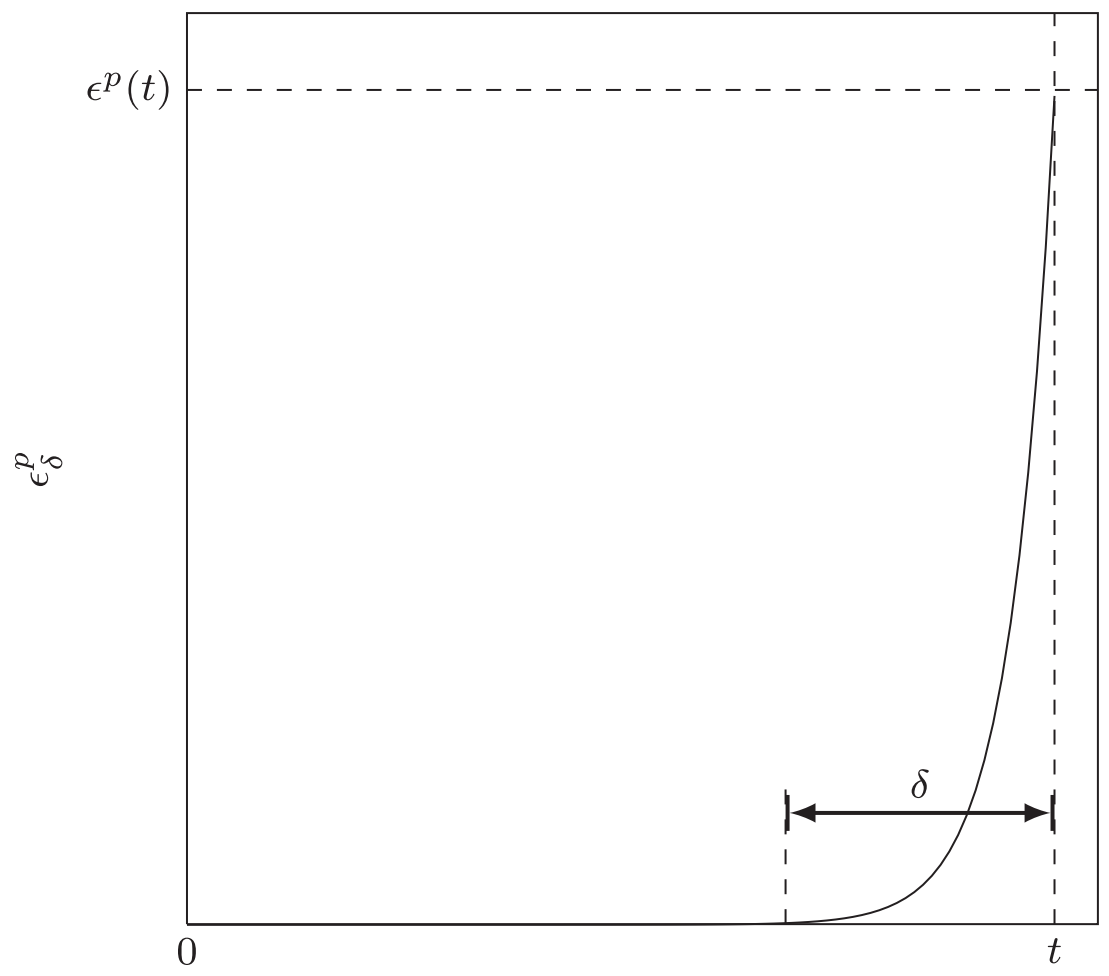

Figure 13. Schematic of the strain history given in Eq. (148).

For a limiting case when $\delta \rightarrow 0$, it can be observed using Eq. (144) that,

$$
\int_{0}^{t} G\left(\epsilon_{\delta}^{p}(y)\right) \dot{\epsilon}_{, k}^{p}(y) d y=G(0) \epsilon_{, k}^{p}(t)=\int_{0}^{t} G(0) \dot{\epsilon}_{, k}^{p}(y) d y
$$

leading to

$$
\int_{0}^{t}\left(G\left(\epsilon_{\delta}^{p}(y)\right)-G(0)\right) \dot{\epsilon}_{, k}^{p} d y=0 .
$$

The above relation is true for any $\dot{\epsilon}_{, k}^{p}(y)$ and hence $G\left(\epsilon_{\delta}^{p}(y)\right)=G(0)=G$ must be a constant, independent of $\epsilon^{p}$. For $G=$ constant, Eq. (147) after integration gives a free energy of the form,

$$
\psi^{p}\left(\epsilon^{p}, \epsilon_{, k}^{p}\right)=\frac{1}{2} G \epsilon_{, k}^{p} \epsilon_{, k}^{p}+\phi\left(\epsilon^{p}\right) .
$$

Consequently the flow rule becomes,

$$
\sigma_{e}=S\left(\epsilon^{p}\right)-G \nabla^{2} \epsilon^{p}
$$

which is exactly same as the flow rule due to Aifantis (1984) in Eq. (109). As shown in Eq. (153) this form can only result when the higher order stress is completely energetic in nature. It can 
also be noticed that a vanishing free energy $(G=0)$ makes the above relation to reduce to a flow rule

conventional in nature.

$$
\sigma_{e}=S\left(\epsilon^{p}\right)
$$

\section{References}

Acharya A and Bassani J L 2000 Lattice incompatibility and a gradient theory of crystal plasticity. J. Mech. Phys. Solids 48(8): 1565-1595 ISSN 0022-5096. doi: 10.1016/S0022-5096(99)00075-7. URL http:// www.sciencedirect.com/science/article/pii/S0022509699000757

Aifantis E C 1984 On the microstructural origin of certain inelastic models. J. Eng. Mater. 106: 326-330

Aifantis K E and Willis J R 2005 The role of interfaces in enhancing the yield strength of composites and polycrystals. J. Mech. Phys. Solids 53(5): 1047-1070 ISSN 0022-5096. doi: 10.1016/j.jmps.2004.12.003. URL http://www.sciencedirect.com/science/article/pii/S002250960500013X

Arsenlis A and Parks D M 1999 Crystallographic aspects of geometrically-necessary and statistically-stored dislocation density. Acta Mater. 47(5): 1597-1611 ISSN 1359-6454. doi: 10.1016/S1359-6454(99) 00020-8. URL http://www.sciencedirect.com/science/article/pii/S1359645499000208

Ashby M F 1970 The deformation of plastically non-homogeneous materials. Philos. Mag. 21: 399-424

Bažant Z and Jirásek M 2002 Nonlocal integral formulations of plasticity and damage: Survey of progress. J. Eng. Mech. 128(11): 1119-1149 doi: 10.1061/(ASCE)0733-9399(2002)128:11(1119)

Bilby B A, Bullough R and Smith E 1955 Continuous distributions of dislocations: A new application of the methods of non-riemannian geometry. Proceedings of the Royal Society of London. Series A, Mathematical and Physical Sciences, 231 (1185): pp. 263-273. ISSN 00804630. URL http://www.jstor. org/stable/99752

Borg U, Niordson C F, Fleck N A and Viggo Tvergaard 2006 A viscoplastic strain gradient analysis of materials with voids or inclusions. Int. J. Solids. Struct. 43: 4906-4916

Budiman A S, Han S M, Greer J R, Tamura N, Patel J R and Nix W D 2008a A search for evidence of strain gradient hardening in au submicron pillars under uniaxial compression using synchrotron $\mathrm{x}$-ray microdiffraction. Acta Mater. 56: 602-608

Budiman A S, Han S M, Greer J R, Tamura N, Patel J R and Nix W D 2008b A search for evidence of strain gradient hardening in au submicron pillars under uniaxial compression using synchrotron $\mathrm{x}$ ray microdiffraction. Acta Mater. 56(3): 602-608 ISSN 1359-6454. doi: 10.1016/j.actamat.2007.10.031. URL http://www.sciencedirect.com/science/article/pii/S1359645407006994

Chakraborty J 2006 Theory of plasticity. Butterworth-Heinemann; 3 edition, UK

Chen Ke, Meng W J, Fanghua Mei, Hiller J and Miller D J 2011 From micro- to nano-scale molding of metals: Size effect during molding of single crystal al with rectangular strip punches. Acta Mater. 59: 1112-1120 doi: 10.1016/j.actamat.2010.10.044

Christian F Niordson 2003 Strain gradient plasticity effects in whisker-reinforced metals. J. Mech. Phys. Solids 51(10): 1863-1883 ISSN 0022-5096. doi: 10.1016/S0022-5096(03)00003-6. URL http://www. sciencedirect.com/science/article/pii/S0022509603000036

Christian F Niordson and John W Hutchinson 2003 Non-uniform plastic deformation of micron scale objects. Int. J. Numer. Meth. Engng. 56: 961-975

Christian F Niordson and Pia Redanz 2004 Size-effects in plane strain sheet-necking. J. Mech. Phys. Solids 52: 2431-2454

Christian F Niordson and Viggo Tvergaard 2006 Size effects on cavitation instabilities. J. Appl. Mech. 73(2): 246-253

Courtney T H Mechanical behaviour of materials. McGraw-Hill, ISBN 0-07-013265-8, 620.11292,C86M

Dimiduk D M, Uchic M D and Parthasarathy TA 2005 Size-affected single-slip behavior of pure nickel microcrystals. Acta Mater. 53(15): 4065-4077 ISSN 1359-6454. doi: 10.1016/j.actamat.2005.05.023. URL http://www.sciencedirect.com/science/article/pii/S1359645405002880 
El-Dasher B S, Adams B L and Rollett A D 2003 Viewpoint: experimental recovery of geometrically necessary dislocation density in polycrystals. Scri. Mater. 48(2): 141-145 ISSN 1359-6462. doi: 10.1016/ S1359-6462(02)00340-8. URL http://www.sciencedirect.com/science/article/pii/S1359646202003408

Ekkehart Kröner 1959 Allgemeine kontinuumstheorie der versetzungen und eigenspannungen. Arch. Rational Mech. Anal. 4: 273-334 ISSN 0003-9527. doi: 10.1007/BF00281393.

Eshelby J D 1956 The continuum theory of lattice defects. volume 3 of Solid State Physics, pages 79-144. Academic Press. doi: 10.1016/S0081-1947(08)60132-0. URL http://www.sciencedirect.com/ science/article/pii/S0081194708601320

Evans A G and Hutchinson J W 2009 A critical assessment of theories of strain gradient plasticity. Acta Mater. 57: 1675-1688

Fleck N A, Muller G M, Ashby M F and Hutchinson J W 1994 Strain gradient plasticity: Theory and experiment. Acta Metall. Mater. 42: 475-487

Fleck N A and Hutchinson J W 1997 Strain gradient plasticity. Adv. Appl. Mech. 33: 295-361

Fleck NA and Hutchinson J W 2001 A reformulation of strain gradient plasticity. J. Mech. Phys. Solids 49(10): 2245-2271 ISSN 0022-5096. doi: 10.1016/S0022-5096(01)00049-7. URL http://www. sciencedirect.com/science/article/pii/S0022509601000497

George E Deiter 1976 Mechanical metallurgy. Mc-Graw Hill

George M Pharr, Erik G Herbert and Yanfei Gao 2010 The indentation size effect: A critical examination of experimental observations and mechanistic interpretations. Ann. Rev. Mater. Res. 40: 271-292

Gerhard A Holzapfel 2000 Nonlinear solid mechanics: A continuum approach for engineering. New York: John Wiley \& Sons, Ltd

Gracio J J 1994 The double effect of grain size on the work hardening behaviour of polycrystalline copper. Scri. Metall. Mater. 31(4): 487-489 ISSN 0956-716X. doi: 10.1016/0956-716X(94)90024-8. URL http://www.sciencedirect.com/science/article/pii/0956716X94900248

Hall E O 1951 The deformation and ageing of mild steel: Iii discussion of results. Proc. Phys. Soc. Sect. B 64(9): 747 URL http://stacks.iop.org/0370-1301/64/i=9/a=303

Jeffrey W Kysar, Yong X Gan, Timothy L Morse, Xi Chen and Milton E Jones 2007 High strain gradient plasticity associated with wedge indentation into face-centered cubic single crystals: Geometrically necessary dislocation densities. J. Mech. Phys. Solids 55(7): 1554-1573 ISSN 0022-5096. doi: 10.1016/ j.jmps.2006.09.009. URL http://www.sciencedirect.com/science/article/pii/S0022509606001657

John W Hutchinson 2012 Generalizing J2 flow theory: Fundamental issues in strain gradient plasticity. Acta Mechanica Sinica 28: 1078-1086 ISSN 0567-7718. doi: 10.1007/s10409-012-0089-4.

Johnson K L 1970 The correlation of indentation experiments. J. Mech. Phys. Solids 18: 115-126

Julia R Greer and William D Nix 2006 Nanoscale gold pillars strengthened through dislocation starvation. Phys. Rev. B 73: 245410 doi: 10.1103/PhysRevB.73.245410.

Kiener D, Pippan R, Motz C and Kreuzer H 2006 Microstructural evolution of the deformed volume beneath microindents in tungsten and copper. Acta Mater. 54(10): 2801-2811 ISSN 1359-6454. doi: 10.1016/j.actamat.2006.02.024. URL http://www.sciencedirect.com/science/article/pii/S135964540600 1455

Kysar JW, Saito Y, Oztop MS, Lee D and Huh WT 2010 Experimental lower bounds on geometrically necessary dislocation density. Int. J. Plasticity 26(8): 1097-1123 ISSN 0749-6419. doi: 10.1016/j.ijplas. 2010.03.009. URL http://www.sciencedirect.com/science/article/pii/S0749641910000574. Special Issue In Honor of Lallit Anand

Ma Q and Clarke D R 1995 Size dependent hardness of silver single crystals. J. Mater. Res. 10: 853-863

Matthew R Begley and John W Hutchinson 1998 The mechanics of size-dependent indentation. J. Mech. Phys. Solids 46: 2049-2068

McElhaney K W, Vlassak J J and Nix W D 1998 Determination of indenter tip geometry and indentation contact area for depth-sensing indentation experiments. J. Mater. Res. 13: 1300-1306

McClintock F A 1968 A criterion for ductile fracture by the growth of holes. J. Appl. Mech. 35(2): 363-371

Michael D Uchic and Dennis M Dimiduk 2005 A methodology to investigate size scale effects in crystalline plasticity using uniaxial compression testing. Mat. Sci. Eng. A-Struct. 268-278 
Michael D Uchic, Dennis M Dimiduk, Robert Wheeler, Paul A Shade and Hamish L Fraser 2006 Application of micro-sample testing to study fundamental aspects of plastic flow. Scri. Mater. 54(5): 759-764 ISSN 1359-6462. doi: 10.1016/j.scriptamat.2005.11.016. URL http://www.sciencedirect.com/science/ article/pii/S1359646205007360

Morton E Gurtin and Lallit Anand 2009 Thermodynamics applied to gradient theories involving the accumulated plastic strain: The theories of aifantis and fleck and hutchinson and their generalization. J. Mech. Phys. Solids 57: 405-421

Morton E Gurtin, Eliot Fried and Lallit Anand 2010 The mechanics and thermodynamics of continua. New York: Cambridge University Press

Motz C, Schobrel T and Pippan R 2005 Mechanical properties of micro-sized copper bending beams machined by the focused ion beam technique. Acta Mater. 53: 4269-4279

Nye J F 1953 Some geometrical relations in dislocated crystals. Acta Metall. Mater.1 doi: 10.1016/0001-6160(53)90054-6

Niordson C F 2008 On higher-order boundary conditions at elasticplastic boundaries in strain-gradient plasticity. Philos. Mag. 88(30-32): 3731-3745 doi: 10.1080/14786430802154823. URL http://www. tandfonline.com/doi/abs/10.1080/14786430802154823

Paolo Cermelli and Morton E Gurtin 2001 On the characterization of geometrically necessary dislocations in finite plasticity. J. Mech. Phys. Solids 49(7): 1539-1568 ISSN 0022-5096. doi: 10.1016/S0022-5096 (00)00084-3. URL http://www.sciencedirect.com/science/article/pii/S0022509600000843

Peter Gudmundson 2004 A unified treatment of strain gradient plasticity. J. Mech. Phys. Solids 52: 13791406

Petch N J 1953 J. Iron Steel Inst. London 173: 25

Pantleon W 2008 Resolving the geometrically necessary dislocation content by conventional electron backscattering diffraction. Scri. Mater. 58(11): 994-997 ISSN 1359-6462. doi:10.1016/j.scriptamat. 2008.01.050. URL http://www.sciencedirect.com/science/article/pii/S1359646208000912

Poole W J, Ashby M F and Fleck N A 1996 Micro-hardness of annealed and work-hardened copper polycrystals. Scri. Mater. 34: 559-564

Rashid K Abu Al-Rub and George Z Voyiadjis 2004 Analytical and experimental determination of the material intrinsic length scale of strain gradient plasticity theory from micro- and nano-indentation experiments. Int. J. Plasticity 20(6): 1139-1182 ISSN 0749-6419. doi: 10.1016/j.ijplas.2003.10.007. URL http://www.sciencedirect.com/science/article/pii/S0749641903001463

Ranjana Saha and William D Nix 2002 Effects of the substrate on the determination of thin film mechanical properties by nanoindentation. Acta Mater. 50: 23-38

Rice J R and Tracey D M 1969 On the ductile enlargement of voids in triaxial stress fields. J. Mech. Phys. Solids 17(3): 201-217 ISSN 0022-5096. doi: 10.1016/0022-5096(69)90033-7. URL http://www. sciencedirect.com/science/article/pii/0022509669900337

Robert Asaro and Vlado Lubarda 2006 Cambridge University Press. URL http://dx.doi.org/10.1017/ CBO9780511755514

Stölken J S and Evans A G 1998 A microbend test method for measuring the plasticity length scale. Acta Mater. 46: 5109-5115

Shu J Y, Fleck N A, Van der Giessen E and Needleman A 2001 Boundary layers in constrained plastic flow: comparison of nonlocal and discrete dislocation plasticity. J. Mech. Phys. Solids 49(6): 1361-1395 ISSN 0022-5096. doi: 10.1016/S0022-5096(00)00074-0. URL http://www.sciencedirect.com/science/article/ pii/S0022509600000740

Swadener J G, George E P and Pharr G M 2002 The correlation of the indentation size effects measured with indenters of various shapes. J. Mech. Phys. Solids 50: 681-694

Tang H, Schwarz KW and Espinosa H D 2007 Dislocation escape-related size effects in single-crystal micropillars under uniaxial compression. Acta Mater. 55(5): 1607-1616 ISSN 1359-6454. doi: 10.1016/ j.actamat.2006.10.021. URL http://www.sciencedirect.com/science/article/pii/S1359645406007464

William D Nix and Huajian Gao 1998 Indentation size effects in crystalline materials: A law for strain gradient plasticity. J. Mech. Phys. Solids 46: 411-425 